\title{
The Surface Wind Influence on the Heat Fluxes Variability on the South Atlantic
}

Douglas Lindemann ( $\sim$ douglasdasilva.lindemann@gmail.com )

Federal University of Pelotas: Universidade Federal de Pelotas https://orcid.org/0000-0002-7503-143X

Alvaro Avila-Diaz

Universidade Federal de Vicosa

\section{Luciano Pezzi}

Instituto Nacional de Pesquisas Espaciais

Jackson Rodrigues

Universidade Federal Fluminense

Rose Ane Freitas

Universidade Federal de Pelotas

Lucas Coelho

Universidade Federal de Pelotas

Marcelo Alonso

Universidade Federal de Pelotas

Wilmar L. Cerón

Universidad del Valle

\section{Research Article}

Keywords: CMIP5, ERA5, heat flux, OAFlux, WIND 1

Posted Date: February 25th, 2021

DOI: https://doi.org/10.21203/rs.3.rs-235260/v1

License: (c) (i) This work is licensed under a Creative Commons Attribution 4.0 International License.

Read Full License 


\title{
THE SURFACE WIND INFLUENCE ON THE HEAT FLUXES VARIABILITY ON THE SOUTH ATLANTIC
}

\author{
Douglas Lindemann ${ }^{1}$, Alvaro Avila-Diaz ${ }^{2}$, Luciano Pezzi ${ }^{3}$, Jackson Rodrigues ${ }^{4}$, Rose Ane Freitas ${ }^{1}$, Lucas Coelho ${ }^{1}$, \\ Marcelo Alonso ${ }^{1}$ and Wilmar L. Cerón ${ }^{5}$ \\ ${ }^{1}$ Department of Meteorology, Universidade Federal de Pelotas, Pelotas, Brazil \\ ${ }^{2}$ Department of Agricultural Engineering, Universidade Federal de Viçosa, Viçosa, Brazil \\ ${ }^{3}$ Laboratory of Ocean and Atmosphere Studies, Earth Observation and Geoinformatics Division, Instituto Nacional de \\ Pesquisas Espaciais, São José dos Campos, Brazil \\ ${ }^{4}$ Education Institute of Angra dos Reis, Universidade Federal Fluminense, Angra dos Reis, Brazil \\ ${ }^{5}$ Department of Geography, Faculty of Humanities, Universidad del Valle, Cali, Colombia \\ Correspondence author: douglasdasilva.lindemann@gmail.com
}

\section{Abstract}

An adequate representation by models and reanalyzes is fundamental since the coverage by observational data on the oceans is still limited. Therefore, this paper aims to evaluate the influence of the wind near the surface on the heat fluxes during the southern winter and summer seasons. Datasets from Coupled Model Intercomparison Project Phase 5 (CMIP5) and reanalyzes were used, in comparison to Objectively Analyzed Air - sea Fluxes (OAFlux) for the South Atlantic Ocean (SAO) during 1980-2005. Results point out an overestimation on the CMIP5 models and reanalyzes to reproduce the heat flux latent and sensible fluxes of SAO, mainly at medium and high latitudes. One possibility may be related the underestimating of surface wind speed, causing an impacts on the heat exchange between ocean and atmosphere. It was also possible to verify that the representation of heat flux, specific humidity, and air and ocean temperatures shows small biases (Mean Bias Error (MBE) to specific humidity ( $\left.\pm 5 \mathrm{~kg}^{\mathrm{kg}} \mathrm{kg}^{-1}\right)$ and sensible heat flux $\left( \pm 10 \mathrm{~W} \cdot \mathrm{m}^{-2}\right)$ ). To the test Root Mean Square Error (RMSE)-observations Standard deviation Ratio (RSR), air temperature values are less than $1{ }^{\circ} \mathrm{C}$, and for the wind with values greater than $2 \mathrm{~m} . \mathrm{s}^{-1}$. There is less precision of CMIP5 models than OAFlux, resulting in low correlation values (between -0.3 and 0.3 ). On the other hand, the reanalyzes show small biases in air and ocean temperatures (between $\pm 1{ }^{\circ} \mathrm{C}$ ) and significant correlations (above 0.9) with the best performances for the NCEP and ERA5.

Keywords: CMIP5, ERA5, heat flux, OAFlux, WIND 


\section{Introduction}

Heat flux on the ocean surface, including turbulence and radiation heat flux, are particularly important in measuring heat at the ocean-atmosphere interface and water vapor transfer (Li et al. 2011). An important mechanism for transporting heat is the water phase change, especially the evaporation that directly results from the latent surface

42 heat flow (LHF), which is an essential part of the hydrological cycle (Trenberth et al. 2009; Rodell et al. 2015). 43 Turbulent surface fluxes have become important in the global climate system. They are the main way the atmosphere exchanges dynamics and thermodynamics properties with the ocean e.g., momentum, heat, moisture, and $\mathrm{CO}_{2}$ 45 (Bourassa et al. 2010).

According to Acevedo et al. (2010), turbulent flux of latent and sensitive heat are one of the ways in which energy is exchanged between the ocean and the atmosphere. Therefore, their correct determination on the oceans is decisive to understand the effects of ocean-atmosphere interactions on the climate (Yu et al. 2011; Pezzi et al. 2005, 2016; Freitas et al. 2019; Leyba et al. 2019). The heat exchange components between ocean and atmosphere include short and long-wave radioactive fluxes, LHF, and sensible heat flux (SHF). As heat fluxes have large interannual and spatial variability (Chou et al. 2004), it is important to observe and obtain a reliable long-term estimate of these fluxes. The spatial and temporal variability of heat fluxes has been explored in previous studies (Bentamy et al. 2003; Yu et al. 2004). The spatial patterns and temporal variability of LHF in the intraseasonal oscillation have been characterized by satellite observations (Grodsky et al. 2009).

Turbulent LHF and SHF are challenging to be measured, especially on ocean surfaces, due to the higher evaporation rate, cloud cover, and precipitable water column when compared to the continents, in addition to the difference in methodologies and data collection (Smith et al. 2010; Pinker et al. 2014; L’Ecuyer et al. 2015; Pezzi et al. 2016; Santini et al. 2020). However, heat fluxes have been estimated using a variety of types of parametrization (Edson et al. 1991; Fairall et al. 1996), considering the differences in average temperature, specific humidity, and wind speed between the ocean surface and the atmospheric layer immediately above.

Such parametrizations form a set of equations used on numerical algorithms (the so-called Bulk Formulas) to estimate LHF and SHF. They were first developed to be used in light to moderate wind conditions and subsequently were modified to include stronger winds (Fairall et al. 2003). For example, Pezzi et al. (2009, 2016) and Santini et al. (2020) have already used heat and momentum fluxes parametrizations with in situ data collected in the Southwest Atlantic Ocean region, in addition to Fairall et al. $(1996,2003)$ for a set of data collected with buoys in the Tropical Pacific Ocean. It is important to note that Pezzi et al. (2016) and Santini et al. (2020) used their Bulk Formula calculation to compare their direct measurements using eddy covariance of heat and momentum fluxes. appropriate network to cover the whole ocean, hindering a more objective analysis, which could provide data sets that would meet the scientific community needs for studying the oceans and climate. Furthermore, to offer an objective view of the annual, seasonal, and inter-annual variability of heat fluxes between the ocean and atmosphere

72 (Yu and Weller, 2007; Yu et al. 2007). However, large data gaps are found, mainly in the South Atlantic Ocean 73 (SAO) studies. 
In addition, SAO is still little studied, when compared to other ocean basins. This region is significantly important for the global climate, especially for the climate of South America, because this is a cyclogenetic area and the oceanic part of the South Atlantic Convergence Zone (Gan and Rao 1991; Hoskins and Hodges 2005; Gramcianinov et al. 2019; Souza and Pezzi, 2020) over warm ocean waters of SAO affecting southeastern South American climate.

According to Dal Piva et al. (2011), warmer waters from the Brazil Current favor creating intense fluxes of latent and sensitive heat on the surface and consequently, the increase of cyclone intensity. Pezzi et al. (2016) suggest the formation of these cyclones occurs in a baroclinic environment and the latent and sensitive heat released by warmer waters affects only their intensity, in contrast to what happens in tropical cyclones, in which LHF and SHF play an essential role for their genesis and development. According to Acevedo et al. (2010), usually, turbulent fluxes over the ocean are determined by a volume ratio, by which sensitive (latent) heat fluxes are proportional to the temperature gradient (specific humidity) between the air and sea surface, between wind magnitudes and a stability coefficient.

An alternative to work around the lack of data on heat fluxes is to use global climate models. In this sense, the numerical models, e.g., ones used on Coupled Model Intercomparison Project Phase 5 (CMIP5) have bulk parametrizations them that are employed to estimate those turbulent fluxes. Zhang et al. (2018) have used an ensemble of models from CMIP5 to evaluate the global ocean latent heat flux and compared them with eleven datasets (reanalyzes, satellites and a combination of both). Their results showed that the mean ensemble condition and the seasonal cycle of LHF match to products (ship-based, satellite-based, blended, and reanalysis), except for a small overestimation over tropical regions and larger biases occurring in coastal regions. A prominent upward trend in the global average LHF is ratified for all LHF products from 1979 to 2005. Despite the strong increase in LHF in CMIP5 models, these increase rates are much weaker than in the data found.

Cao et al. (2015) evaluated the mean climatological condition, the seasonal variation, and the long-term trend of LHF in historical series of fourteen CMIP5 models and Objectively Analyzed air-sea Fluxes (OAFlux) data for the Pacific Ocean during the 1979-2005 period. Results showed that the models could capture the climatological LHF features; however, the amplitudes are generally overestimated. Besides, the LHF seasonal variations simulated by the models compare well with observations but with overestimated amplitudes. These authors point out that the wind speed is primarily underestimated and can cause theses biases in the CMIP5 models. The regression analysis carried out by Cao et al. (2015) has indicated that the diversity among the models may come from the simulated diversity of the sea surface temperature (SST) and specific humidity of the atmosphere near the surface. Comparing the LHF observed long-term trends and wind speed trends are underestimate. In contrast, SST trends and specific air humidity are grossly overestimated, which may be the origin of model deviations in the LHF trend reproduction.

Based on the considerations made previously, we identified the low number of studies done on, and we identified the low number of studies done on the turbulent heat fluxes over the SAO. We identified the need to study

108 the relation of heat fluxes and the variables that influence these fluxes over the SAO. To accomplish this proposition

109 we chose an ensemble of climate models from CMIP5 and reanalyzes data, covering the period from 19802005

110 during the summer and winter seasons. 
112 area, datasets used, and methodologies applied; Section 3 - Results obtained by average and spatial statistical 113 performances; Section 4 - Discussion of results; and Section 5 - Final considerations.

\section{Material and Methods}

In this section we present details about the study area and its subdivisions, the data sets used and the statistical tests applied to evaluate the performance of the reanalyzis and the CMIP5 models.

\subsection{Study area and data set}

There are spatial and temporal variations in heat fluxes among the different datasets used, and according to results found in the literature (Pezzi et al. 2016; Leyba et al. 2019). As a way to work around such troubles, the area of interest in this study is the South Atlantic Ocean (SAO) $\left(75^{\circ} \mathrm{W}, 25^{\circ} \mathrm{E} ; 60^{\circ} \mathrm{S}, 5^{\circ} \mathrm{N}\right)$ as shown in Fig. 1 . The area was split into three regions: (a) Southwestern Subtropical Atlantic (SW-SSA, $70^{\circ}$ and $21^{\circ} \mathrm{W} ; 55^{\circ}$ and $25^{\circ} \mathrm{S}$ ); (b) Southeastern Subtropical Atlantic (SE-SSA, $20^{\circ} \mathrm{W}, 20^{\circ} \mathrm{E} ; 55^{\circ}$ and $25^{\circ} \mathrm{S}$ ); and (c) Tropical South Atlantic (TSA, $45^{\circ}$ and $18^{\circ} \mathrm{W} ; 24^{\circ}$ and $0^{\circ} \mathrm{S}$ ) (Fig. 1). Also considering the region atmospheric characteristics, two seasonal periods were selected, the southern summer which is comprised by December, January, and February (DJF) months and the southern winter which is comprised by June, July, and August (JJA) months.

Figure 1. area of interest in this study is the South Atlantic Ocean (SAO). The area was split into three regions: Southwestern Subtropical Atlantic (SW-SSA); Southeastern Subtropical Atlantic (SE-SSA); and Tropical South Atlantic (TSA).

The datasets involve the models from CMIP5 for the simulations of the Historical of the World Climate Research Program (Taylor et al. 2012). The data of the models that are part of CMIP5 were obtained through: https:// esgf-node.llnl.gov/projects/cmip5/. Details of each model that will compose the Multi-Model Ensemble (MME) are shown in Table 1.

Table 1. CMIP5 models and reanalyzes comprising the study with their respective names, abbreviations, resolution grid, and reference

Climate reanalyzes were used to assess the performance of the CMIP5 models (individually and as MME). The reanalysis of OAFlux (Yu and Weller, 2007; Yu et al. 2008) was used as the observed datasets (http://oaflux.whoi.edu/), as it is a reanalysis already evaluated and compared to previously observed data (Yu et al. 2008; Cao et al. 2015; Bentamy et al. 2017). Other reanalysis were also used, one of which is the ERA5 (Hersbach et al. 2020), that is part of European Centre for Medium-Range. The other reanalysis are: Weather Forecasts National Centers for Environmental Prediction and the National Center for Atmospheric Research (NCEP/NCAR1) 
151 (http://jra.kishou.go.jp/JRA-25/index_en.html) reanalyzes were also included in this study to compare with OAFlux

152 model (Table 1). All CMIP5 models and reanalyzes are interpolated at $1.0^{\circ}$ x $1.0^{\circ}$ longitude/latitude horizontal 153 resolution.

154 In addition to the heat fluxes (LHF and SHF), other variables that may influence the variation of flows over the OAS were also analyzed, they are: air temperature at $2 \mathrm{~m}$ above the displacement height (T2M), sea surface temperature (SST), specific air humidity (Q), and wind speed at $10 \mathrm{~m}$ high (WIND).

\subsection{Performance metrics}

159

The OAFlux was used as an observed dataset to evaluate the statistical performance of the climate reanalyzes, 13 CMIP5 models, and their MME. We use three performance metrics: The Mean Bias Error (MBE), the Ratio of Root Mean Square Error to the observations standard deviation of the observation (RSR), and the Pearson correlation coefficient (CORR). These statistical parameters are widely used to assess the ability of the CMIP5 models and reanalyzes to simulate climatic variables (Akhter et al. 2017; You et al. 2017; Avila-Diaz et al. 2020a, 2020b). More details of each method can also be found in Moriasi et al. (2007).

The first test applied was the MBE, which is related to the model's ability to adjust and represent the data considered as observed, that is, the bias is the difference between the expected model prediction value and the actual value intended to be represented in Equation (1):

172 in which the overbar represented the mean predicted $\left({ }^{P}\right.$ ) and mean observed $\left({ }^{\bar{O}}\right.$ ) by the model or reanalyse. 173 Using MBE, it is generally intended to point out an average polarization model, that is, a value above or below the 174 mean. The MBE can also point out useful information, but it must be interpreted with caution, since it is inconsistently related to the magnitude of the typical error (Fekete et al. 2004; Willmott and Matsuura, 2005). RMSE is of particular interest because it is one of the most widely reported and misinterpreted statistical error measurements in the climate and environmental literature (Singh et al. 2004; Willmott and Matsuura, 2005). Although it is commonly accepted that the lower the RMSE, the better the model performance, Singh et al. (2004) published a guideline to qualify what is considered a low RMSE according to the standard deviation of the observations. Moriasi et al. (2007) followed the Singh et al. (2004) recommendations and developed an evaluating statistical models called the RMSE-observations standard deviation ratio (RSR), it is calculated as the ratio between the RMSE and the standard deviation of the measured data as shown below in Equation (2): 


$$
R S R=\frac{R M S E}{S T D E V_{O b s}}=\frac{\sqrt{\left(P_{i}-O_{i}\right)^{2}}}{\sqrt{\left(O_{i}-\bar{O}_{i}\right)^{2}}}
$$

According to Moriasi et al. (2007), RSR incorporates the benefits of the statistical error index and includes a scale/normalization factor so that the resulting statistic and reported values could be applied to different constituents. The RSR ranges from the ideal value of 0 , which indicates zero RMSE or residual variation.

Finally, CORR was used, which describes the degree of collinearity between simulated and observed data. CORR ranges from -1 to 1 and can be considered a degree index of linear relationship between observed data and simulated data. If $C O R R=0$, there is no linear relationship. If $C O R R=1$ or $C O R R=-1$, there is a perfect positive or negative linear relationship (Santhi et al. 2001; van Liew et al. 2003). CORR is obtained by dividing the covariance of the model and the data observed by its standard deviations (Equation 3):

$$
\text { CORR }=\frac{\sum_{i=1}^{n}\left(P_{i}-\bar{P}_{i}\right)\left(O_{i}-\bar{O}_{i}\right)}{\sqrt{\sum_{i=1}^{n}\left(O_{i}-\bar{O}_{i}\right)} \sqrt{\sum_{i=1}^{n}\left(P_{i}-\bar{P}_{i}\right)}}
$$

Spatial analyzes of the MBE were also performed for the heat flows (LHF and SHF) and WIND, in order to better assess which specific regions that the reanalysis and the MME can have a good (or bad) performance. The data sets' capability was assessed to represent the dominant patterns of LHF and WIND variability through the Empirical Orothogonal Functions (EOF) analysis. EOF calculations are based on the covariance matrix and carried out separately for boreal summer and winter. A more comprehensive explanation of the EOF can be found in Lorenz (1956) and Wilks (2011).

\section{Results}

\subsection{Statistical tests}

The performance of reanalysis and CMIP5 models will be demonstrated under different aspects: an analysis related to the performance of these sets for heat flux and other variables that may interfere with the spatial variability of these flows. And later, a spatial statistical analysis for SAO.

The SAO was divided into three regions and analyzed in summer (DJF) and winter (JJA) from 1980 to 2005. To assess the individual performance, the climate dataset for each variable over the different regions. Furthermore, to analyze heat fluxes (LHF and SHF), variables that directly influence such flux were also evaluated. Considering the heat fluxes from the three SAO regions, it is observed that most of the models from CMIP5 and MME overestimate LHF and SHF values for both analyzed periods (DJF and JJA). The MBE highest values 
(values above $10 \mathrm{~W} \cdot \mathrm{m}^{-2}$ ) for LHF are found in the TSA region (Fig. 2, first and fourth columns), indicating a positive

218 bias in the models from CMIP5. These models have better performance for the other two SAO regions, mainly during the winter season (JJA), but mostly keeping a positive bias.

Reanalyzes also shows this behavior observed in the CMIP5 models with high MBE values, overestimating the LHF (values above $5 \mathrm{~W} \cdot \mathrm{m}^{-2}$, Fig. 2a). ERA5 was the exception, which underestimated (values below $-5 \mathrm{~W} \cdot \mathrm{m}^{-2}$ ) the LHF values in most conditions, except for the SE-SSA and SW-SSA regions during DJF, when the reanalysis shows small positive values (Fig. 2a).

Overestimations also occur in most CMIP5 models when the SHF is analyzed but with lower values (values above $1 \mathrm{~W} \cdot \mathrm{m}^{-2}$ ) when compared with the LHF. Only two models (FGOALS-s2 and MIROC5) indicated an underestimation (values below $-1 \mathrm{~W} \cdot \mathrm{m}^{-2}$ ) prevalence for SHF. Reanalyzes showed a better behavior for SHF with lower and overestimated MBE values (values above $0.05 \mathrm{~W} \cdot \mathrm{m}^{-2}$ ); in the same way that ERA5 previously underestimated LHF values, the CFSR pointed out values well below for SHF than the values observed (values below $-5 \mathrm{~W} \cdot \mathrm{m}^{-2}$ ). It is also important to note that only JRA-25 has not pointed out MBE values underestimated in the SE-SSA region during JJA $\left(1 \mathrm{~W} \cdot \mathrm{m}^{-2}\right)$.

An alternative way to analyze the variability of these datasets for the LHF consists of evaluating their performance for other variables that directly interfere in the heat flux. In this case, were analyzed WIND, Q, T2M, and SST. Thus, it is noteworthy that the CMIP5 models and the reanalyzes tend to underestimate WIND $\left(-5 \mathrm{~m}^{-1} \mathrm{~s}^{-1}\right)$ in practically all SAO sectors during DJF and JJA (Fig. 2a).

The largest underestimations (values above $-5 \mathrm{~m} \cdot \mathrm{s}^{-1}$ ) of the models and reanalyzes for WIND occurred in the SE-SSA and SW-SSA regions in both seasons (DJF and JJA). Underestimations (-1 m.s. ${ }^{-1}$ ) also occurred in the TSA, but with less intensity (Fig. 2a).

Figure 2. Statistical tests for the variables under analysis for each SAO region (TSA, SE-SSA, and SW-SSA) during the two seasonal periods (DJF and JJA); for the thirteen models from CMIP5, the Ensemble (MME) and the four reanalyzes compared with the observational dataset - OAFlux: a) BIAS (top panel); b) the RSR (central panel); and c) the CORR (bottom panel). The black line on the CORR indicates that the correlation is significant at 95\%.

When calculating the MBE for Q, an underestimation (values between -1 and $1 \mathrm{~kg}^{\mathrm{kg}}{ }^{-1}$ ) was found among most models and reanalyzes (Fig. 2a). However, the Q values are lower when compared with the values found in WIND. The highest underestimations (above $5 \mathrm{~kg} \mathrm{~kg}^{-1}$ ) occurred during the DJF in the TSA region, and most models and reanalyzes showed values that ranged between -1.0 to $1.0 \mathrm{~kg} \cdot \mathrm{kg}^{-1}$ in the other regions (Fig. 2a). It is also important to highlight the cases of the BESM-OA2-5 and MRI-ESM1 that have pointed out a different pattern from most models, a pattern of overestimation in practically all regions and periods under analysis. In contrast, most models have pointed out an underestimation pattern for Q.

The NCEP and JRA-25 showed good behavior concerning OAFlux for Q with MBE variations from 0.05 to -0.05 in all regions during the southern summer and winter. The CFSR was the one that pointed out weak

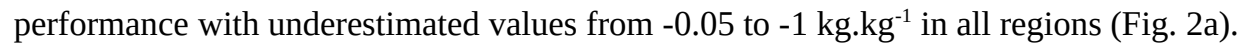

The models showed quite distinct patterns for SST (Fig. 2a). Whereas some models indicated an overestimating behavior of this variable (BESM-OA2-5, CNRM-CM5-2, GISS-E2-R, INCM4, and MRI-ESM1), 
other models pointed out to an underestimation of SST (CanESM2, CSIRO-Mk3-6-0, IPSL-CM5A-MR, MIROC5, and NorESM1-ME) with values between $-1{ }^{\circ} \mathrm{C}$ and $-5^{\circ} \mathrm{C}$.

MME and reanalyzes also indicated an underestimation pattern in SST, with values around $-1{ }^{\circ} \mathrm{C}$. It is important to note that the TSA and SW-SSA regions were the ones that had the highest underestimations during DJF and JJA periods. Only the NCEP indicates small overestimations during JJA in the TSA and SW-SSA regions (Fig. 2a).

In general, T2M showed an underestimation (values between -1.0 and $1.0^{\circ} \mathrm{C}$ ) pattern of datasets related to most CMIP5 models compared with the OAFlux dataset (see last eight columns of Fig. 2a). SST and this characteristic are also found in MME and reanalyzes. Variations from -1.0 to $1.0^{\circ} \mathrm{C}$ occur in most conditions, except for CSIRO-Mk3-6-0 and IPSL-CM5A-MR models with the values exceeding $-5^{\circ} \mathrm{C}$ (Fig. 2a).

It should be noted that most of the sets present a greater positive and negative bias for LHF and WIND, respectively. However, only the MBE evaluation cannot guarantee that a given dataset satisfactorily represents the variations in the observed data. Thus, the RSR test is used to analyze the representation behavior of the datasets.

As seen in the MBE, the RSR metric points out that the models and reanalyzes have the lowest LHF and WIND behavior. It is notorious that the CMIP5 models present a better behavior when compared to the reanaly zes in the heat fluxes (LHF and SHF) in both seasonal periods (Fig. 2b, central panel). The reanalyzes stand out only in the SW-SSA region during DJF and JJA concerning the CMIP5 models.

Whereas the models stood out to the reanalyzes in flux, the same behavior was not verified in WIND (Fig. $2 \mathrm{~b}$ ), a variable in which the datasets showed the worst results than OAFlux SAO high latitudes ( SE-SSA and SWSSA) during DJF and JJA. The sets show better behavior only in the equatorial region (TSA).

The best behavior of the datasets for the RSR was due to the variables Q and T2M, in which the values were mostly close to 0 (Fig. 2b). This behavior was also observed in SST, except for some specific cases such as MIROC5 and JRA-25. Whereas MIROC5 pointed out better results for the SW-SSA region in seasonal periods, JRA-25 showed better behavior for the SE-SSA region.

In terms of correlation test (CORR; Fig. 2c), CMIP5 models behave poorly for heat fluxes (LHF and SHF), as most CORR values were not statistically significant. On the other hand, the reanalyzes presented satisfactory results with significant correlations at $95 \%$ in practically all situations. The equatorial region (TSA) was the one that showed the lowest (values below 0.3) CORR values between reanalyzes and OAFlux for SHF during DJF.

When analyzing the behavior of the sets for WIND, the models were keeping the pattern previously verified with the heat flux. Still, the reanalyzes also have pointed out poor behavior (Fig. 2c). The best results (with significance) were collected in the regions of TSA and SE-SSA during DJF, and the significant results were concentrated in the TSA and SW-SSA regions during JJA. Among the reanalyzes, the worst behavior was for JRA25 , not indicating significance in any regions.

The behavior was similar in the other variables (Q, SST, and T2M), whose models were showing low CORR values in relation to OAFlux and significant CORR for reanalyzes (Fig. 2c). CORR vary around 0.6 for Q, except in the SW-SSA region during JJA, in which the CORR values reached values of 0.95 for the CFSR and NCEP. 
NCEP also showed the best behavior for SST and T2M with CORR values above 0.9 for all regions and periods (Fig. 2c). In the specific case of SST, the lowest CORR values were found in the SE-SSA during the DJF and JJA for all reanalyzes, whereas the highest CORR values were identified for T2M, mainly in the NCEP - as mentioned earlier - and ERA5 during JJA.

In general, the reanalyzes produce relatively better results than the models from CMIP5. We found that NCEP followed by ERA5 and CFSR is the best resource to simulate the variables studied and supply possible no data observed in the SAO. On the other hand, the best relative results are found by MME, NorESM1-ME, FGOALSs2, and GISS-E2-R datasets.

\subsection{Spatial Variations}

The reanalyzes and MME of CMIP5 models were analyzed to determine which subregions have the greatest spatial variability concerning the OAFlux data.

The atmospheric and oceanic characteristics determine the SAO regions with the highest/lowest heat fluxes and energy exchange between the two systems. The seasonal variation of the high-pressure system South Atlantic subtropical high (SASH) in the southeast SAO influences the strengthening/weakening of the southeast trade winds close to the equatorial region. During the southern summer period (DJF), the trade winds are weaker than the northeast trade winds (Sun et al. 2017; Reboita et al. 2019). This pattern associated with the southern Equatorial

311 Current involves higher LHF values near the coastal region from the Brazilian Northeast (Fig. 3a), according to the LHF data, agreeing with the results found by Leyba et al. (2019).

Figure 3. Latent heat flux (LHF) in W.m ${ }^{-2}$ from 1980 to 2005, DJF (a-f) and JJA (g-m): a-g) OAFLux climatology; MBE calculation from datasets and OAFlux: b-h) ERA5; c-i) NCEP, d-j) CFSR, e-k) JRA-25; and f-l) MME

Another region with intense energy exchanges between the atmosphere and the oceanic surface is located at the southwestern region of the SAO in the Brazil-Malvinas Confluence (BMC). In this region, the two ocean currents meet with different characteristics: Brazil Current with a hot and saline current, and the Malvinas Current with a cold and less saline current. This combination favors BMC as one of the most energetic regions on the planet with intense heat and energy exchanges between the ocean and the atmosphere (Josey et al. 2008; Combes and Matano, 2014).

The LHF climatology of the OAFlux points out that the lowest LHF values are found in regions with colder T2M and SST, as at high latitudes south of $40^{\circ} \mathrm{S}$, in the resurgence on the African Coast region, in the Malvinas Current up to the direction of the BMC region (Fig. 3a). According to Pinker et al. (2014), these minimum LHF values are found mainly in regions with advection of cold waters, determining characteristics of the regions above mentioned.

When comparing the MBE of OAFLux with the other datasets, it is noteworthy that small differences occur between ERA5 (Fig. 3b) and OAFlux with negative values from -10 to $-20 \mathrm{~W} \cdot \mathrm{m}^{-2}$ in the equatorial region over the Guinea Current and close to the northeastern Brazilian coast. This behavior justifies the high MBE of ERA5 (Fig. 2a) for the LHF in TSA during DJF. A small area with positive values is found over the resurgence region in the Benguela Current. 
The NCEP (Fig. 3c) and CFSR (Fig. 3d) reanalyzes show similar behavior with MBE positive values in the tropical region, ranging from 10 to $40 \mathrm{~W} \cdot \mathrm{m}^{-2}$, extending along the South America Coast, suggesting a greater intensity of the Brazil Current when compared with OAFlux. The MBE negative values are found for the NCEP case, the same is not observed in the CFSR. In fact, the positive values extend across most of the subtropical Atlantic (SW-SSA and SE-SSA regions) in the CFSR, and in the case of NCEP the positive values are restricted only to the southeastern coast of South America, the rest of the subtropical Atlantic has negative values.

Still in the region of the TSA, positive values are observed to the south of $5^{\circ} \mathrm{S}$, while to the north of $5^{\circ} \mathrm{S}$ and the equator they present negative values that extend from the coast of Africa to $10^{\circ} \mathrm{W}$.

Even higher MBE values are found for JRA-25 (Fig. 3e) and MME (Fig. 3f). The largest overestimations are located near the SAO tropical region, in the case of JRA-25, extending to the Brazilian Coast (values from 10 to $40 \mathrm{~W} \cdot \mathrm{m}^{-2}$ ), as previously found in the NCEP and CFSR. There are overestimations in the equatorial region in both sets with values close to $50 \mathrm{~W} . \mathrm{m}^{-2}$ compared whit observation (Fig. 2a). High MBE values were also found in the Benguela resurgence region by all reanalyzes and MME.

BMC is another region overly sensitive to such variations, where the JRA-25 pointed out a more intense Brazil Current, as found in the previous cases of the NCEP and CFSR. The MME showed a dipole pattern with positive anomalies at the north BMC and negative anomalies at the south region.

The main differences found in the LHF among the OAFLux and the other datasets are concentrated mainly in three regions of the SAO: (a) in the resurgence on the Africa West Coast; (b) in the equatorial and tropical regions; and (c) in the BMC (Fig. 3). Many variables can influence theses changes, such as wind speed, cloud cover, precipitable water column, specific moisture content present in the atmosphere, and temperature differences between the ocean and the atmosphere (Pinker et al. 2014).

During the southern winter period (JJA), the main atmospheric characteristics in the SAO are centered on the westward displacement of the SASH, on the strengthening of the resurgence on the Africa West Coast, and the weakening of the trade winds at the southwest (Sun et al. 2017; Reboita et al. 2019). It should be stressed that the weakening/strengthening of the Brazil Current/Malvinas Current along the Brazilian Coast/Argentine Coast, favoring the BMC being moved further north (Combes and Matano, 2014).

The set of factors above mentioned can favor so that the most considerable flux of LHF (Fig. 3g) are concentrated in the SAO tropical region, mainly near the northeast and southeast regions of Brazil, where LHF values reach close to $200 \mathrm{~W} \cdot \mathrm{m}^{-2}$ in the SAO tropical region during JJA, as indicated by the climatology of OAFlux.

Other regions with positive LHF values are associated with the presence of trade winds from the northeast (equatorial region) and the region of the Brazil Current (South of Brazil, Uruguay, and Argentina) and in the south of the African continent in the region of Agulhas Current (Fig. 3g). On the other hand, LHF negative values are found mainly in regions of cooler waters as in the southern latitudes of $40^{\circ} \mathrm{S}$, in resurgence regions on the Africa West Coast, and the Malvinas Current in South America Southeast Coast.

The predominance of LHF positive values during DJF (Fig. 3a) and JJA (Fig. 3g) at the north of $40^{\circ} \mathrm{S}$ indicates that there is a significant loss of heat from the ocean towards the atmosphere during the two periods under analysis (Liu and Curry, 2006). The highest values are found during JJA due to the greater moisture contrast between 
the ocean surface and the atmosphere, and the higher wind speed near the surface. According to Bourassa et al. (2010), this found pattern occurs because the LHF and SHF at the ocean-atmosphere interface oscillate in magnitude throughout the year with SHF changing its sign. In most regions, the ocean stores heat during the summer and releases it in the winter (Pinker et al. 2014).

When evaluating the behavior among the other sets concerning the OAFlux for the MBE test, there are small underestimations of ERA5 (Fig. 3h) in the equatorial region (less than $-20 \mathrm{~W} . \mathrm{m}^{-2}$ ). These values are close to zero in the tropical region (up to $20^{\circ} \mathrm{S}$ ) with a small overestimation $\left(10 \mathrm{~W} \cdot \mathrm{m}^{-2}\right)$ on the Africa West Coast. On the other hand, the NCEP (Fig. 3i) presents a strong contrast between underestimation/overestimation in the equatorial/tropical region, with values reaching $\pm 30 \mathrm{~W} \cdot \mathrm{m}^{-2}$. Analyzing the southern regions of SAO, strong underestimations are found in the Agulhas Current and the BMC, whose pattern can favor the significant underestimations (Fig. 2a).

The other sets showed a similar pattern with overestimations (values reaching $50 \mathrm{~W} \cdot \mathrm{m}^{-2}$ ) centered mainly in the tropical region (from $30^{\circ} \mathrm{S}$ to $0^{\circ}$ ). In the case of CFSR (Fig. 3j), the highest MBE values are located near the Brazilian Northeast Coast and in the southeast trade winds region, indicating a possible weakening of the S ASH. In addition to this region, positive values are also found on the warm side of the BMC $\left(20 \mathrm{~W}^{-2} \mathrm{~m}^{-2}\right.$, where the Brazil current usually meets the Malvinas Current during the JJA period.

JRA-25 (Fig. 3k) and MME (Fig. 3l) show overestimations of LHF mainly from $0^{\circ}$ to $40^{\circ} \mathrm{S}$, with higher values in the tropical region (JRA-25) of a resurgence on the African coast (MME) and in the BMC region (both). Regions with underestimations $\left(-20 \mathrm{~W} \cdot \mathrm{m}^{-2}\right)$ are found in the Agulhas Current and BMC. A possible justification for this is that the underestimation is related to the grid resolution of these sets since the two regions above mentioned are highlighted by intense heat exchange processes between ocean and atmosphere in spatial scales smaller than the grids of the sets (Small et al. 2008).

Byrne et al. (2016) used regional simulations with a coupled high-resolution ocean-atmosphere model in the South Atlantic to show that their thermodynamic interactions with the atmosphere can energize the mesoscale oceanic characteristics and the vortices in particular. Oceanic vortices in the presence of a large-scale wind gradient provide a mesoscale channel to transfer energy to the ocean due to SST anomalies, affecting the wind field above them. According to Byrne et al. (2016), the above process is responsible for up to $10 \%$ of the kinetic energy of the oceanic mesoscale vortices in the South Atlantic, and these conditions occur mainly in the Antarctic Ocean north of the Polar Front.

According to the OAFlux climatology, the highest SHF values during DJF are concentrated in regions with intense SST and T2M gradients (Fig. 4a), such as the Agulhas Current regions (values above $30 \mathrm{~W} \cdot \mathrm{m}^{-2}$ ) and in the BMC (values close to $9 \mathrm{~W} . \mathrm{m}^{-2}$ ). In the SAO equatorial and tropical regions, the values range from 3 to $9 \mathrm{~W} . \mathrm{m}^{-2}$, pointing out that the warmer ocean transfers energy to the colder atmosphere. The reverse process occurs from $40^{\circ} \mathrm{S}$ towards high latitudes, where the SHF values range from -3 to $-9 \mathrm{~W} \cdot \mathrm{m}^{-2}$; that is, the atmosphere gives heat to the colder ocean surface. The most significant negative values of SHF are concentrated mainly in the Malvinas Current region, meeting the Brazil Current, characterized as a hot current, resulting in an intense dynamic process in the BMC. 
Figure 4. Latent heat flux (LHF), in $\mathrm{W} \cdot \mathrm{m}^{-2}$, from 1980 to 2005, DJF (a-f) and JJA (g-m): a-g) climatology of OAFLux; calculation of MBE between datasets and OAFlux: b-h) ERA5; c-i) NCEP, d-j) CFSR, e-k) JRA-25; and f-I) MME

When the behavior of reanalyses and MME with OAFlux for SHF was evaluated through the MBE test (Fig. 2a), strong underestimations of the NCEP and CFSR for the SE-SSA and SW-SSA regions were identified during DJF, whereas ERA5 and MME were showing values close to zero, pointing out a good performance. On the other hand, the JRA-25 has overestimated the SHF values in the TSA.

When spatially evaluating the performance of the sets for the SHF, underestimations of up to $-10 \mathrm{~W} \cdot \mathrm{m}^{-2}$ were identified in the SE-SSA region for the NCEP (Fig. 4c), whereas overestimations were occurring in the tropical region near the coast of Africa and coast of Brazil. Such SHF underestimations in the SE-SSA were also found in the CFSR (Fig. 4d), and this pattern extends to the SW-SSA region, justifying the result showed in Fig. 2a. The NCEP also has pointed out regions with underestimations (SE-SSA and SW-SSA), but there is also an overestimation at the north BMC, which can reduce the result when calculating the average of the area.

Small MBE values are found in ERA5 for SHF during DJF (Fig. 4b), not exceeding \pm 5 W.m ${ }^{-2}$, with overestimation/underestimation occurring along the Brazilian Coast, BMC, and at $50^{\circ} \mathrm{S}$ (Agulhas Current). Although the MME may indicate good behavior (Fig. 2a), it is possible to identify areas with values above $10 \mathrm{~W} \cdot \mathrm{m}^{-2}$ (Fig. 4f), pointing out an overestimation in Africa's resurgence region West Coast and also in the SAO high latitudes. Regions with SHF underestimations concerning OAFlux were found in the BMC and Agulhas Current regions.

Overestimations occurred in the equatorial and tropical regions by JRA-25 (Fig. 4e) with values reaching 20 W. $\mathrm{m}^{-2}$ at the Africa West Coast. This pattern is following the result found in this study (Fig. 2a). The lower overestimation at the SW-SSA (Fig. 2a) can be justified by the existing contrast with overestimation at the SW-SSA North. However, a pattern of underestimation was identified at the SW-SSA South.

The southern thermal gradient at the SAO during JJA favors the positive SHF values identified at $30^{\circ} \mathrm{S}$ (Fig. 4g), where SHF values exceed $30 \mathrm{~W} \cdot \mathrm{m}^{-2}$ at $50^{\circ} \mathrm{S}$, during JJA. According to Morrison et al. (2016), who used simulations from the global climate model, SST is on average hotter than the atmosphere in most of the ocean in the Southern Hemisphere high latitudes; therefore, SHF cools the ocean. The salient peak of heat absorption in the Southern Ocean from $50^{\circ}$ to $60^{\circ} \mathrm{S}$ is dominated by a positive anomaly (reduction in cooling) in the SHF component, related to the decreasing temperature difference at the ocean-atmosphere interface. Also, the presence of deep-water resurgence keeps a relatively constant SST at the Southern Ocean, where the atmosphere above warms up more quickly than the ocean, increasing the temperature gradient in the ocean-atmosphere interface. In the tropical region, SHF values are close to zero, pointing out that heat exchanges between the atmosphere and ocean practically cancel each other over the period.

MBE results for SHF during JJA are like those seen during DJF. However, it is worth mentioning that major

442 underestimations occur in medium and high latitudes of SAO, mainly for ERA5 (Fig. 5h), NCEP (Fig. 5i), and CFSR 
(Fig. 5j), reflecting the results shown in Fig. 2a. Meanwhile, for these same reanalyses, small MBE or dipole patterns are found in the tropical and equatorial region, contributing to the MBE low oscillations in the TSA region.

The JRA-25 has pointed out overestimation in the tropical region with values above $20 \mathrm{~W} \cdot \mathrm{m}^{-2}$ at the Africa West Coast. At medium and high latitudes, significant contrasts were identified, and in the case of MME (Fig. 4l).

In the two heat flux analyzed so far (LHF and SHF), the spatial patterns identified in the MBE for reanalyses and the MME (Fig. 4l) are very similar in both seasonal periods under analysis, which may be a consequence of the general circulation of the atmosphere and the contrast between the ocean and the atmosphere in both seasonal periods. Besides, each SAO subregion has a particular characteristic, making it challenging to homogenize heat flux for it.

Atmospheric circulation near the surface plays an essential role in the heat exchange between the atmosphere and the ocean. Thus, the adequate representation of WIND over SAO is fundamental to understand the heat flow variations in different seasonal periods. During DJF, the WIND climatology (Fig. 5a) calculated with OAFlux data have pointed out more intense winds at $35^{\circ} \mathrm{S}$, associated with west winds, reaching values from 9 to 10 $\mathrm{m} \cdot \mathrm{s}^{-1}$.

In addition to the westerly winds at medium and high latitudes, the weakest/strong SASH can make the Southeast/Northeast trade winds weaker/stronger and stand out (Sun et al. 2017; Reboita et al. 2019). This condition favors the appearance of intense LHF values in the tropical region near the Brazil Northeastern Coast (Fig. 3a). Less intense winds over the tropical and SAO equatorial region favor increased evaporation and, consequently, LHF values.

Evaluating the performance of reanalyses and MME by the MBE statistical test, underestimating of the sets concerning OAFlux is noted, especially at the SAO medium and high latitudes. The highest underestimations have occurred in the SW-SSA with the highest values in ERA5 (Fig. 5b), NCEP (Fig. 5c), and JRA-25 (Fig. 5e) with values from $-3 \mathrm{~ms}^{-1}$ to below $-5 \mathrm{~ms}^{-1}$. CFSR (Fig. 5d) and MME (Fig. 5e) also pointing out underestimations but with a smaller spatial pattern.

In addition to the SW-SSA, underestimations were also found in the SE-SSA, mainly in the southern region of the South African continent, whose values were below $-5 \mathrm{~m} \cdot \mathrm{s}^{-1}$. MBE values were close to zero at the latitudes from $20^{\circ}$ to $5^{\circ} \mathrm{S}$, pointing out a better representation of the sets, including the MME with low overestimations (Figs. $5 a$ until 5f). The sets came again to underestimate WIND in the tropical region where trade winds operate, but with lower values compared to SW-SSA, whose values have not exceeded $-3 \mathrm{~m} \cdot \mathrm{s}^{-1}$ in the TSA region, thus, justifying the best performance found by the sets in the data spatial average (Fig. 2a).

During JJA, the westerly winds at medium latitudes become more intense (Fig. 5g), reaching values above $11 \mathrm{~m} . \mathrm{s}^{-1}$. In this period, SASH moves west and becomes more intense, increasing its occupation area on SAO (Sun et al. 2017; Reboita et al. 2019). The more intense southern gradient winds favor the westerly winds, and the southeast trade winds become more intense, these overlapping the northeast trade winds.

Figure 5. Wind speed (WIND), in m.s ${ }^{-1}$, from 1980 to 2005, DJF (a-f) and JJA (g-m): a-g) OAFLux climatology; calculation of MBE between datasets and OAFlux: b-h) ERA5; c-i) NCEP, d-j) CFSR, e-k) JRA-25; and f-l) MME. 
The performance of the sets during JJA is like that found during DJF, with even greater underestimations in medium and high latitudes. The best performances in the tropical region were from MME (Fig. 5l) and CFSR (Fig. 5j), with values close to zero. However, ERA5 (Fig. 5h), NCEP (Fig. 5i), and JRA-25 (Fig. 5k) were indicated as small values of MBE when compared with OAFlux.

\subsection{Empirical Orthogonal Function (EOF) to analyze the climate patterns}

One way to represent the main characteristics of a particular variable over a region is by the EOF. The principal components (PC) of the reanalyses and MME of the models for LHF are displayed in Figs. 6a-6f (DJF) and Figs. 6g-6l (JJA). Fig. 6a shows the PC1 of OAFlux (variance of 19.55\%), in which positive values are centered in the most intense regions in terms of dynamics and energy in the SAO, namely, the Agulhas Current and the BMC. There are also positive values in the tropical region, from $10^{\circ} \mathrm{S}$ to $0^{\circ}$. Meanwhile, negative values are present in the SAO central region from $45^{\circ}$ to $30^{\circ} \mathrm{S}$, matching the average position of the SASH during DJF.

The EOF1 of ERA5 (Fig. 6b) keeps the pattern found for OAFlux but with a larger area of positive values in the tropical region and absence of negative values at the north of the Equator (variance of 19.23\%). The EOF1 of the NCEP (Fig. 6c) and JRA-25 (Fig. 6e) show a similar pattern, prevailing the positive values over the SAO, except at the north of the Equator and east of the equatorial Atlantic Cold Tongue. However, these negative values occupy a larger area, according to the JRA-25. These reanalyzes also do not point out the negative values at the SAO central region found in the OAFlux. And it is the reanalyses that present the greatest variances, when compared to OAFlux (24.92\% and 22.34\%, respectively). In turn, the EOF1 of CFSR (Fig. 6d) and MME (Fig. 6f) show an adverse pattern compared to OAFlux, with negative values in the tropical region and part of the SE-SSA prevalent, mainly in MME. Positive values are found in part of the SW-SSA, and the CFSR is pointing out positive values in the Agulhas Current region. And these reanalyzes indicated the smallest variances, when compared with OAFlux (18.85\% and $15.46 \%$, respectively).

Figure 6. Seasonal spatial loading patterns of the first LHF mode computed for the 1980-2005 period in the south Atlantic Ocean, for DJF (a-f) and JJA (g-m): a-g) OAFLux; b-h) ERA5; $\mathbf{c - i}$ ) NCEP, d-j) CFSR, e-k) JRA-25; and fl) MME.

The JJA period (Figs. 6g-6l) has achieved more harmonious EOF1 than the DJF period, with a wide prevalence of positive anomalies over the SAO. In contrast, small areas with negative anomalies are found in the western region of the TSA. This region with negative anomalies occupies a smaller area in ERA5 (Fig. 6h) and MME (Fig. 6l), with $17.17 \%$ and 19.62\%, respectively. In the NCEP (Fig. 6i) and CFSR (Fig. 6j), the predominance of negative anomalies at the west of the TSA occurs. However, the negative anomalies pointed out by the JRA-25 (Fig. 6k) are concentrated at the east of the TSA. The variance of these three reanalysis is $22.73 \%, 17.95 \%$ and $31.49 \%$, respectively. It is important to emphasize the predominance of positive anomalies of OAFlux (Fig. 6g) over SW-SSA, whereas the other sets indicate negative anomalies between the Brazil Southern Coast and BMC (15.89\% 
variance). The highest values were found in ERA5 (Fig. 6h) and MME (Fig. 6l), and the lowest values were found in JRA-25 and NCEP.

LHF is directly influenced by WIND, as the presence of wind near the ocean-atmosphere interface influences the evaporation and heat exchange process between the two environments. During DJF, the main characteristics of WIND over SAO were concentrated in the presence of a high pressure center in the SW-SSA near the Argentina Coast, in addition to a SASH displaced to the east and the more intense northeast trade winds, moving the Intertropical Convergence Zone (ITCZ) further south from its climatological position, as seen in EOF1 of ERA5 (Fig. 7b).

The EOF1 of the wind speed showed positive values from $50^{\circ}$ to $35^{\circ} \mathrm{S}$, associated with the west winds over the SAO, and the negative values from $50^{\circ} \mathrm{S}$ towards the high latitudes are related to westerly winds and north-south winds in the tropical and equatorial regions. WIND is more intense in OAFlux (Fig. 7a) in the TSA region, prevalent across SAO, except for the east wind region around $40^{\circ} \mathrm{S}$, with a variance of $28.55 \%$.

The NCEP (Fig. 7c) is the set that most closely resembles the conditions of the OAFlux but with more intense east winds, just as noted in ERA5. The EOF1 behavior of the vectors in the NCEP is also following the standard indicated in ERA5, and with a variance closer to OAFlux. While NCEP obtained $29.77 \%$ of variance, ERA5 indicated 31.28\%. The CFSR (Fig. 7d) and JRA-25 (Fig. 7e) also followed the characteristics found in ERA5, such as the high pressure center in SW-SSA, the SASH further east, and the presence of the northeast trade winds. The only difference related to previous patterns is positive values in the resurgence region on the Africa Coast, and higher variance values, 31.07\% for the CFSR and 33.13\% for the JRA-25.

Figure 7. First Principal Component of EOF for WIND, from 1980 to 2005, for DJF (a-f) and JJA (g-m): a-g) OAFLux; b-h) ERA5; c-i) NCEP, d-j) CFSR, e-k) JRA-25; and f-l) MME.

The MME (Fig. 7f) has pointed out a good performance in WIND compared to the reanalyzes, but not representing the high pressure center in SW-SSA, where the trade winds were less intense and the SASH was displaced to further south of SAO, in addition, the variance value (59.35\%) was very different when compared to the other sets for the DJF period.

The pattern on the SAO during JJA was focused mainly on the intensification and displacement to the SASH west, in addition to the change in the behavior of the trade winds, when the winds of the southeast quadrant become prevalent in the equatorial region, as seen in EOF1 of ERA5 (Fig. 7h). The positive values of the WIND were concentrated in the SASH peripheral region, and the negative values were located near the center of the high pressure.

As discussed in the DJF, ERA5 was pointing out a smaller area representing WIND in the SAO tropical region, compared to OAFlux (Fig. 7g), in addition to the values being less intense. From $45^{\circ} \mathrm{S}$, the WIND values of ERA5 (Fig. 7h) were more intense than those of OAFlux. There is a region with negative values close to the Equator in OAFlux, probably related to trade winds pattern, a situation not found in ERA5. As occurred in DJF, the NCEP (Fig. 7i) has pointed out a spatial and value pattern closer to OAFlux, even though SASH is represented further south. 
Whereas the CFSR (Fig. 7j) indicated a WIND pattern like OAFlux, the JRA-25 (Fig. 7k) was pointing out a behavior like ERA5. However, the EOF1 of WIND (represented by the vectors) in both reanalyzes was not representing the position of SASH very well. Besides, the CFSR pointed out the entry of more intense east winds into the Agulhas Current in the SE-SSA. It is also important to note that both reanalyses pointed out the prevalence of the southeastern trade winds in the equatorial region. As noted in the DJF, the MME could not accurately pinpoint the trade winds (Fig. 7l) and the winds in the tropical region. Also, SASH is further north than the other sets. The variances of the other sets were slightly lower when compared to OAFlux (21.54\%), the lowest value (20.01\%) was for CFSR and the closest to JRA-25 (20.93\%). The MME variance (27.23\%) was above OAFlux, however, not as discrepant as in the case of DJF.

An acceptable representation of the WIND conditions on the SAO is essential for the set under analysis to have a good representation of the heat flux over the ocean, mainly the LHF. It is possible to see which set represents the best when calculating the correlation between the PC1 of the heat flux (LHF and SHF) with the PC1 of the WIND.

There was a wide prevalence of significant negative correlations between the PC1 of the heat flux and the WIND, except for the CFSR (Table 2). The highest correlations were found in OAFlux (-0.51), in JRA-25 (-0.49), and ERA5 (-0.46) for LHF, whereas the highest values were found for MME (-0.88), JRA-25 (-0.57), and NCEP (0.56) concerning SHF. These values reinforce the results found (Fig. 2c). For example, the JRA-25 was the reanalysis that indicated higher CORR values during DJF than OAFlux, despite pointing out an overestimation pattern of LHF and presenting higher values of RSR in the SW-SSA and the SE-SSA regions. On the other hand, ERA5 has presented a better performance in MBE and RSR tests than JRA-25.

Table 2. Correlations between the first PC1 of LHF and SHF with the first PC of WIND. Underlined values are significant at $95 \%$.

The correlations of PC1 of SHF also reinforce the results (Fig. 2a), since the MME, NCEP, and JRA-25 were the sets that have acquired the best performances in the MBE. On the other hand, whereas the MME has achieved better results for the RSR, the NCEP and JRA-25 have shown better results in the CORR.

During JJA, all sets showed significant correlations between PC1 of LHF and WIND, and the highest values were indicated by NCEP (0.74), CFSR (0.61), and OAFlux (0.58). The NCEP and CFSR were the reanalyzes that pointed out the best MBE indices, in addition to the best RSR and CORR in the SW-SSA region (Fig. 2c), whereas to PCI of SHF, only OAFlux showed a significant correlation with PC1 of WIND with PC1 of SHF, pointing out that

\section{Discussion}

By the MBE test, results exposed in the previous section indicated that, in most cases, there was a tendency to overestimate LHF from the analyzed sets (models and reanalyzes) when compared to the OAFlux. The greatest overestimations occur in the TSA, mainly during the southern winter (JJA) (Fig. 2a). This pattern was reinforced 
when analyzing the spatial characteristics of the sets (Fig. 3), and the reanalyzes and MME were pointing out overestimations in the tropical and equatorial regions, except for ERA5 and NCEP.

The highest overestimations found during JJA may be associated with evaporation because, in all ocean basins, the turbulent transfer of heat is dominated by the latent heating of evapotranspiration, according to L'Ecuyer et al. (2015). In large ocean basins, minimums of evaporation during summer and peaks in winter reinforce the annual cycle of solar radiation in the oceans, causing large seasonal reversals in the net energy exchanged between the oceans and the overlying atmosphere. Furthermore, residues in the annual net heat exchange in individual basins are generally less than $10 \%$ of the seasonal cycle range.

The results above exposed are in agreement with the results obtained by Gao et al. (2013). They stated that the highest LHF values are in the peripheral regions of the ITCZ and also from $15^{\circ} \mathrm{S}$ to $40^{\circ} \mathrm{S}$, when analyzing LHF trends at the global level. According to Leyba et al. (2019), the positive trend of SST can trigger an increase in the differences between T2M and SST, leading to higher LHF and SST. According to these authors, the most prominent variations between T2M and SST were found during JJA and were enhanced by WIND (Pinker et al. 2014).

In the MBE test, the best performances for LHF of ERA5 and NCEP are in agreement with the results found by Feng and $\mathrm{Li}$ (2006), who stated that the reanalyzes results are similar to satellite results from 1988 to 2000 . However, the authors also have found qualitative differences among the reanalyzes; the LHF variations of ERA are near the satellite data in the tropics and medium latitudes, and the NCEP showed a better performance in the other regions.

On the other hand, the significant overestimations found in the Benguela resurgence region are an inverse pattern to that found by Cabos et al. (2017), who used a model with different parameterizations to represent the SASH; they stated that the strong biases found in the Benguela's resurgence region during DJF are associated with the representation of SST and the weaker SASH. This pattern weakens the winds over the southeast region of the tropical Atlantic, deepens the thermocline, and prevents the resurgence of colder waters.

Overestimations also occur in SHF but lower than LHF; however, this pattern was expected because less SHF exchange occurs in oceanic regions. However, there is also a prevalence of overestimation for SHF, except for some sets (FGOALS-s2, MIROC5, ERA5, and CFSR) (Fig. 2a).

Despite a better performance by the models comprising the CMIP5 in the RSR test (Fig. 2b), the reanalyzes have provided a better response with significant CORR for both heat flux in both periods. Only the TSA region during DJF showed more deficient results. Despite these limitations, the sets represented the main characteristics of heat exchanges between both environments in the SAO. Previous studies point out that the greatest differences in heat flux occur in resurgence regions during the winter, mainly due to cloud cover representation by models or reanalyses (Xu et al. 2014; Valdivieso et al. 2017). On the other hand, the deepest thermocline and the atmospheric flux are probably the primary sources of bias during JJA, with variations in the received solar radiation and cloudiness pointing out a secondary effect, according to Cabos et al. (2017).

Specific regions of the SAO also deserve to be highlighted, as in the regions of Agulhas Current and BMC, where intense exchanges of energy between ocean and atmosphere occur, in addition to significant dynamic processes. Josey et al. (2008) explain that this occurs because the net heat flux is higher over the westerly boundary 
currents at medium latitudes, where the warmer waters transported from the Equator to the poles are cooled and evaporated by contact with cold, dry air masses that are usually transported by the westerly winds of subtropical atmospheric gyres.

According to Nkwinkwa et al. (2019), the highest values of LHF are found during JJA in the region of Agulhas Current (Fig. 3g). Small et al. (2008) also highlight that SHF and LHF on the surface increase significantly over the hot side of the Agulhas Current, due to the winds blowing from regions where the water is colder to places where the water is warmer, generating destabilization in the boundary layer over the warmer water, increasing the vertical turbulent mixture, which deepens the boundary layer over the warmer water compared to the colder water.

The BMC region is also characterized by being a region with one of the biggest increases in the heat flux trend in the SAO (Leyba et al. 2019). This factor can contribute to the strong gradients of the heat flux found (Fig. 3 and Fig. 4) with intense overestimations and underestimations to be the result of the regional dynamics, as Acevedo et al. (2010) demonstrate that high LHF values from the ocean dominate an energy partition to the atmosphere on the warm side of the BMC and the cold side under prefrontal conditions. After the frontal passage, the estimates made by the authors suggest that the cold side can become a moisture sink with LHF directed from the atmosphere to the ocean. The estimates also indicated that the cold side acts as a sink for the heat flow at all times, thus removing heat from the atmosphere above it. The LHF on the cold side tends to be directed upwards, but they have a much smaller magnitude than those estimated on the hot side.

The substantial underestimation of WIND over SW-SSA (Fig. 5) pointed out that all sets can directly impact the representation of LHF in the region. When analyzing data found in the BMC region, Pezzi et al. (2005; 2009) stated that a strong SST gradient in the region modulates the atmospheric boundary layer, and the highest flux values are found on the hot side of the confluence. In turn, these more significant fluxes are associated with greater differences between the temperatures of the ocean-atmosphere and wind speeds occurring in this region. This characteristic is striking, where the atmospheric-ocean boundary layer is unstable, and the flux changes are intense. As the reanalyzes and the MME to a lesser extent can represent the variations of T2M and SST in the region with a certain degree of reliability (Fig. 2), it is possible to conclude that the deficient representation of WIND is the main responsible for the overestimation of flux heat in the SW-SSA.

In addition to these regions, the tropical region influenced by trade winds also points out deficiencies in heat flux representations. Valdivieso et al. (2017) state that tropical oceans show heat gain, and subtropics and high latitudes show heat loss. This pattern (Fig. 4a) with regions of high values of LHF comprise the equatorial region, influenced by the more intense trade winds of the northeast and the region at the extreme south of the African continent where the Agulhas Current is responsible for transporting warmer waters from the Indian Ocean. Xu et al. (2014) also determined that this region on the Africa West Coast is a problem for the models comprising the CMIP5 since the models have a positive short-wave radiation bias in agreement with the models' deficiency simulating lowlevel clouds. This error in representing the shortwave radiation is overcome by greater errors in the simulated turbulent surface heat and long-wave radiation flux, resulting in excessive heat loss by the ocean.

Even though the CMIP5 models and the reanalyzes represent different groups, and the most complex ones are the second set with more adjusted interactions and lower parametrization, Liang and Yu (2016) highlight that the 
products are presenting good performance even with deficiencies to represent flux in the region between the 669 Southern Ocean and the SAO. According to the authors, the results generated can provide divergent trends and values about the heat content in the ocean because these products are used as input data in numerical simulations.

The performance of the CMIP5 models and the reanalyzes related to the heat flux over the SAO is directly

672 influenced by other atmospheric variables. Among them, WIND deserves more emphasis, as it is responsible for

673 providing conditions that can produce different characteristics between the ocean surface and the atmosphere just

674 above. It was clear that there is a very robust of underestimating the sets concerning OAFlux, for both periods under 675 analysis (DJF and JJA) and mainly for the medium and high latitude regions (SE-SSA and S W-SSA) (Figs. 3 and 6).

676 The main differences found in the monthly and annual averages of the LHF over the oceans may be associated with

677 the performance deficiencies of Q, and mainly, of the WIND in the datasets used (Feng and Li, 2006; Smith et al. 678 2010).

Disharmonious differences between OAFlux and specific sets of heat flux during DJF and JJA were verified (Figs. 3 and 4). These values can be justified by problems in estimating flux between the atmosphere and the ocean 681 using satellite data and reanalyzes for the Southern Ocean. Swart et al. (2019) stated that these problems might be 682 due to insufficient observations, particularly in winter, and the need to determine the net exchange as the sum of four 683 individual components (LHF, SHF, short wave radiation, and long-wave radiation). The authors also evaluated the 684 spread of the net heat flux among atmospheric reanalyses products, which showed regional differences of up to 60

$685 \mathrm{~W} \cdot \mathrm{m}^{-2}$. These differences persist when the zonal average is calculated. It is important to note that values from 40 to $68660 \mathrm{~W} \cdot \mathrm{m}^{-2}$ were also found among OAFlux, JRA-25, and MME for LHF in the tropical region during DJF and JJA.

687 Additionally, Kumar et al. (2017) found that the winds are always originated at sea in tropical regions. The 688 authors also pointed out that large-scale winds blow from regions with cold SST towards regions with warmer SST 689 and, therefore, the surface air is more humid and colder than SST. This fact keeps a constant temperature gradient 690 throughout the year, resulting in a constant Q gradient throughout the year.

The weak representation of the atmospheric circulation near the surface (Fig. 7) hinders a better 692 performance of the sets under analysis. Particularly during DJF, the SASH spatial pattern responds to local thermal 693 forcing, ocean cooling in the eastern sector of the ocean basin and heating in the adjacent continental areas, 694 providing an increase in SHF. This cooling in the eastern sector is mainly due to the radiative loss produced by 695 stratus and stratocumulus clouds over the ocean. These clouds create obstacles for solar radiation to reach the 696 surface, which does not favor increasing SST. Also, the winds from the south quadrant cause advection of cold 697 waters in this region (Miyasaka and Nakamura, 2010; Reboita et al. 2019).

698 Reboita et al. (2019) state that SASH has a more zonal characteristic, and its center displaced to the west 699 during JJA, unlike DJF, which is more circular and displaced to the east. Other authors have also found an 700 intensification and expansion of SASH in recent decades and that this pattern can influence the increase in SST at 701 SAO (Cherchi et al. 2018). This SASH behavior also leads to an intensification of the pressure gradient, causing an 702 increase in WIND and intensifying the Brazil Current. Consequently, the transport to the south of warmer SST is 703 increased (Reboita et al. 2019; Leyba et al. 2019). Certain sets (CFSR, JRA-25, and MME) still have difficulties in 704 keeping this WIND pattern (Fig. 5), causing problems in the behavior of the LHF and SHF representation (Fig. 2). 
As previously mentioned, the problem in the representation of WIND is a decisive factor in heat flux. It is important to note that, in the case of SAO, there are regions with atmospheric characteristics that vary greatly among seasonal periods, such as the Agulhas Current and the BMC region, with intense and variable atmospheric circulations.

When analyzing datasets with models and reanalyzes, Valdivieso et al. (2017) have found differences in models and reanalyzes for the LHF representation, precisely due to deficiencies in the WIND representation. Furthermore, using satellite data, Gao et al. (2013) also have found that the temporal changes in the LHF are associated with variations in the wind near the surface and the difference in specific humidity between the ocean and atmosphere. Analyzing data from reanalyzes and satellites, Nkwinkwa et al. (2019) also have stated that the sets representing best the LHF in the Agulhas Current region were the ones with the best performance in representing WIND and Q.

During JJA, SST has played an important role. Tokinaga et al. (2005) included a detailed climatology of ocean-atmosphere coupling patterns, where a positive correlation between SST and sea surface wind intensity was noted. Leyba et al. (2019), when analyzing the period from 1982 to 2015, also have found a close relationship in the $\mathrm{Q}$ and WIND trend, stating that the differences among these variables and the influence of SST on the SW-SSA region of SAO favor to explain the behavior and the tendency of LHF during the JJA period, being the SST the primary variable responsible for the LHF behavior. The authors state that the SASH intensification favors an increase in WIND, intensifying, in turn, the west current, leading to an increase in SST and, therefore, to the intensification of heat flux in the region, justifying the significant positive correlation values found between PC1 of LHF and PC1 of WIND during JJA period (Table 2).

In addition to the particularities related to the SAO typical atmospheric circulation system above discussed, it is needed to highlight the interference of oceanic vortices, which are very common over the SAO and which provide a strong mixture in the surface layer of the ocean, driving, in turn, a strong mixture on the surface, a decrease in SST, and an increase in SHF, as explained by Villas Bôas et al. (2015). These vortices can help understand the major regional differences in SHF, SW-SSA, and SE-SSA during the JJA period (Fig. 4). According to Freitas et al. (2019), regions with strong SST gradients, for example, BMC, provide higher SHF values, which may be associated with the greater baroclinity of the atmosphere over SW-SSA, and thus favoring so that the highest values can occur during JJA period (Fig. 4).

The biggest performance variations between CMIP5 models and reanalyzes are in the CORR of T2M and SST, compared to OAFlux. Despite the overall underestimation of the sets with some models with clearer underestimations and good performance for the RSR test, the CORR on the models have achieved too low a rate, and

736 the reanalyzes have pointed out significant CORR for both variables (Fig. 2c). Similar behavior was found in Q but with reanalyzes pointing out lower significant CORR.

According to Valdivieso et al. (2017), heat flux between the ocean and the atmosphere is composed of short and long-wave radiation terms and turbulent heat flux (sensitive and latent) calculated by the bulk formulas, and both

740 long-wave radiation and turbulent flux are dependent significantly on SST. That is why it is important to note that 741 the relationship between LHF and SST is pointing out that regions with an increase/decrease in SST favor a 
greater/less heat transfer between the ocean and the atmosphere just above. Besides, significant correlations between SST average monthly anomalies and turbulent heat flux in particular ocean regions, specifically close to border currents, in the Southern Ocean and close to the Equator, are pointing out that these ocean regions are driving atmospheric circulation through SST anomalies with the correlations of medium latitudes only becoming noticeable in oceanic models with $1^{\circ}$ resolution. This fact suggests that errors in the SST modeled or in the surface flow and its variability can cause systematic bias in the models (Kirtman et al. 2012; Roberts et al. 2016).

WIND plays an essential role in this process, helping to transfer new air parcels to those areas with higher SST, keeping the transfer of energy, or reducing this heat transport from the ocean to the atmosphere in regions with colder SST (Yu, 2007). However, the weaker winds over SW-SSA and SE-SSA inhibit the process described above (Fig. 5). Also, a good SST representation with the models is important. Performing a global analysis of the LHF sensitivity to variations in SST focused on tropical regions, Kumar et al. (2017) have found that the sensitivity of variables related directly to LHF (WIND and Q) and SST give rise to dynamic (WIND) and thermodynamic (Q) feedbacks, and that this interaction favors making LHF sensitive to SST mainly in the tropics, where the increase in SST causes changes in the WIND (Q), resulting in a decrease/increase in LHF.

The EOFs calculated in the sets for the LHF (Fig. 6) show spatial patterns matching the PC1 during both periods under analysis (DJF and JJA). Liu et al. (2011) also have found similar patterns when calculating EOFs through reanalyzes for the Southern Ocean; according to the authors, one reason for this good performance is the coupling between the atmosphere and the ocean in the reanalyzes.

However, it is important to emphasize that the representation of the PC1 of WIND in calculating EOF (Fig. 7) is pointing out some variations among the sets, such as a correct location and intensity of the S ASH, as well as the wind belts in medium latitudes, being this last factor the most problematic factor during JJA period. According to Liu et al. (2011), the strong winds (magnitude and frequency) occurring in medium and high latitudes in the Southern Hemisphere can exceed the speeds for which the algorithms were created, mainly for equatorial and tropical regions. These more intense winds also provide an extremely weak oceanic and atmospheric stratification in medium and high latitudes of the SAO, resulting in a deeper limit layer, changing the limits of existing stability parametrizations.

Yu (2007) also have calculated EOF to evaluate the role played by WIND on evaporation in the oceans, suggesting a dominant role for WIND. In which the hypothesis is that the wind causes an impact in evaporation in two ways; one way is direct, when WIND boosts greater evaporation, taking water vapor to regions further away from the evaporative surface, allowing the moisture gradients at the ocean-atmosphere interface to be restored; the second way is indirect, when the intensified WIND strengthens the subtropical gyre, driving, in turn, greater heat transport by the western currents in the oceans, heating the SST in these regions and providing more evaporation by increasing the moisture gradients between the ocean and the atmosphere.

The reasoning exposed by Yu (2007) previously support the results found in the CFSR, JRA-25, and MME (Figs. 2 and 3), in which LHF overestimations occur mainly in TSA, where WIND underestimations are lower. The northeast trade winds also favor a WIND intensification in the region, a higher evaporation rate, and a consequent increase in LHF (Fig. 7). Also, the representation of the high pressure center in SW-SSA can directly influence the 
amount of cold air from high latitudes to be directed to medium latitudes. In return, the inflow of air masses and ocean surface currents to be displaced towards high latitudes.

\section{Conclusions}

This paper intended to present the variations of the LHF and SHF heat flux over the SAO and the main variables that can impact the variability of these fluxes, as air temperature at 2 meters, sea surface temperature, specific air humidity, and wind speed at $10 \mathrm{~m}$ high. A seasonal analysis (DJF and JJA) was carried out for the period from 1980 to 2005. Data from climatic models comprising the CMIP5 and reanalyzes were used and later compared with the OAFlux.

Despite the difference between the models and reanalyzes regarding atmospheric interactions and parametrizations, both sets showed a pattern of the LHF and SHF overestimation over the SAO. However, the SHF was less intense. This behavior is directly related to the WIND underestimation, mainly in the middle and high latitude regions ( SW-SSA and SE-SSA).

Small underestimations were found in the other variables (Q, T2M, and SST) and reasonable accuracy of the sets by the RSR test, except for WIND over the SW-SSA and SE-SSA regions, with the worst performances during the southern winter (JJA).

One way to analyze the performance of the data sets is through the application of EOF (PC1), in this way it was possible to indicate that ERA5 and NCEP obtained the best spatial representations and in the variance value for the LHF, when compared with the OAFlux. The other groups had difficulties in representing the main spatial features in the SAO.

But it is in the calculation of EOF for WIND that the differences in spatial representation were more distinct when compared with OAFlux, such as the correct location and intensity of SASH, as well as of the wind belts in mid latitudes, the latter being the most problematic factor during the JJA period. ERA5, NCEP and CFSR are the sets with the best performance. For WIND, the variances were very close to the OAFlux values.

The main difference between the models and the reanalyzes was apparent in the correlation. In contrast, the models pointed out weak correlations, the reanalyzes showed significant correlations, mainly for T2M and SST, suggesting a better coupling between both systems. This pattern suggests that the models have a low degree of reliability, as they point out low correlation values, although varying slightly compared to the average.

It should be stressed that the primary deficiencies in the representations of heat flux are associated with regions with continuous wind occurrence, as in the trade winds in TSA and around SASH. It is also important to highlight that regions with intense temperature gradients and dynamic processes such as the Benguela resurgence, the Agulhas Current, and the BMC also have low representativeness on the sets with problems the representation of cloud cover and the grid spatial resolution.

Finally, despite the advance and improvement of dynamic and physical representations by models and reanalyzes, the lack of greater coverage of data found on SAO becomes an even greater challenge for better 
816 the heat flux representation problems in the SAO region.

\section{Acknowledgments}

819 The first author would like to thank the Coordination for the Improvement of Higher Education Personnel (CAPES),

820 for their financial support through n. 23110.029260/2018-52. The eighth author was supported by the Universidad

821 del Valle (Cali-Colombia).

822

\section{Declarations}

\section{Funding}

825 Coordination for the Improvement of Higher Education Personnel (CAPES), for their financial support through $\mathrm{n}$.

$82623110.029260 / 2018-52$.

\section{Conflicts of interest/Competing interests}

828 Not applicable

\section{Availability of data and material}

830 Not applicable

831 Code availability

832 Not applicable

\section{References}

Acevedo O, Pezzi, L, Souza R, Anabor V, Degrazia G (2010) Atmospheric boundary layer adjustment to the 837 synoptic cycleat the Brazil-Malvinas Confluence, South Atlantic Ocean. J Geophys Res 115: D22107. 838 doi:10.1029/2009JD013785.

839 Adachi Y, Yukimoto S, Deushi M, Obata A, Nakano H et al (2013) Basic performance of a new earth system model 840 of the Meteorological Research Institute (MRI-ESM1). Papers in Meteorology and Climatology 64: 1-19. 841 https://doi.org/10.2467/mripapers.64.1.

842 Akhter J, Das L, Deb A (2017) CMIP5 ensemble-based spatial rainfall projection over homogeneous zones of India. 843 Clim Dynam 49: 1885-1916. https://doi.org/10.1007/s00382-016-3409-8.

844 Avila-Diaz A, Abrahão G, Justino F, Torres R, Wilson A (2020a) Extreme climate indices in Brazil: evaluation of 845 downscaled earth system models at high horizontal resolution. Clim Dynam 54: 5065-5088. 846 https://doi.org/10.1007/s00382-020-05272-9.

847 Avila-Diaz A, Benezoli V, Justino F, Torres R. Wilson A (2020b) Assessing current and future trends of climate 848 extremes across Brazil based on reanalyses and earth system model projections. Clim Dynam 55: 1403-1426. https:// 849 doi.org/10.1007/s00382-020-05333-z.

850 Bao Q, Lin P, Zhou T et al (2013) The Flexible Global Ocean-Atmosphere-Land System Model, Spectral Version 2: 851 FGOALS-s2. Adv Atmos Sci 30: 561-576. https://doi.org/10.1007/s00376-012-2113-9.

852 Bentamy A, Katsaros K, Mestas-Nuñez A, Drennan W, Forde E, Roquet H (2003) Satellite Estimates of Wind Speed 853 and Latent Heat Flux over the Global Oceans. J Climate 16: 637-656. https://doi.org/10.1175/1520854 0442(2003)016\%3C0637:SEOWSA\%3E2.0.CO;2. 
Bentamy A, Piollé J, Grouazel A, Danielson R, Gulev S et al (2017) Review and assessment of latent and sensible heat flux accuracy over the global oceans. Remote Sens Environ 201: 196-218. https://doi.org/10.1016/j.rse.2017.08.016.

858 Bentsen M, Bethke I, Debernard J, Iversen T, Kirkevag A et al (2013) The Norwegian Earth System Model, 859 NorESM1-M - Part 1: Description and basic evaluation of the physical climate. Geosci Model Dev 6: 687-720. 860 https://doi.org/10.5194/gmd-6-687-2013.

861 Bourassa M, Gille S, Jackson D, Roberts J. Wick G (2010) Ocean Winds and Turbulent Air-Sea Fluxes Inferred 862 From Remote Sensing. Oceanography 23: 36-51. https://doi.org/10.5670/oceanog.2010.04

863 Byrne D, Münnich M, Frenger I. Gruber N (2016) Mesoscale atmosphere ocean coupling enhances the transfer of 864 wind energy into the ocean. Nat Commun 7: 11867. doi: 10.1038/ncomms11867.

865 Cabos W, Sein D, Pinto J, Fink A, Koldunov N et al (2017) The South Atlantic Anticyclone as a key player for the 866 representation of the tropical Atlantic climate in coupled climate models. Clim Dynam 48: 4051-4069. 867 https://doi.org/10.1007/s00382-016-3319-9.

868 Capistrano V, Nobre P, Tedeschi R, Silva J, Bottino M et al (2018) Overview of climate change in the BESM-OA2.5 869 climate model. Geosci Model Dev, https://doi.org/10.5194/gmd-2018-209.

870 Cao N, Ren B, Zheng J (2015) Evaluation of CMIP5 Climate Models in Simulating 1979-2005 Oceanic Latent Heat 871 Flux over the Pacific. Adv Atmos Sci 32: 1603-1616. https://doi.org/10.1007/s00376-015-5016-8.

872 Cerón W, Kayano M, Andreoli R et al (2021) Recent intensification of extreme precipitation events in the La Plata 873 Basin in Southern South America (1981-2018). Atmos Res 249. https://doi.org/10.1016/j.atmosres.2020.105299.

874 Cherchi A, Ambrizzi T, Behera S, Freitas A, Morioka Y, Zhou T (2018) The response of subtropical highs to climate 875 change. Curr Clim Change Rep 4: 371-382. doi: https://doi.org/10.1007/s40641-018-0114-1.

876 Chou S, Chou M, Chan P, Lin P, Wang K (2015) Tropical warm pool surface heat budgets and temperature: 877 contrasts between 1997/98 El Niño and 1998/99 La Niña. J Climate 17: 1845-1858. https://doi.org/10.1175/1520878 0442(2004)017\%3C1845:TWPSHB\%3E2.0.CO;2.

879 Chylek P, Li J, Dubey M, Wang M, Lesins G (2011) Observed and model simulated $20^{\text {th }}$ century Arctic temperature 880 variability: Canadian Earth System Model CanESM2. Atmos Chem Phys 11: 22893-22907. 881 https://doi.org/10.5194/acpd-11-22893-2011.

882 Combes V, Matano R (2014) Trends in the Brazil/Malvinas Confluence region. Geophys Res Lett 41: 8971-8977. 883 https://doi.org/10.1002/2014GL062523.

884 Dal Piva E, Gan M, Moscati M (2011) The role of latent and sensible heat fluxes in an explosive cyclogenesis over 885 South America. J Meteorol Soc Jpn 89: 1-27. https://doi.org/10.2151/jmsj.2011-604.

886 Díaz A, Aceituno P (2003) Atmospheric circulation anomalies during episodes of enhanced and reduced convective 887 cloudiness over Uruguay. J Clim 16: 3171-3185. https://doi.org/10.1175/1520888 0442(2003)016<3171:ACADEO>2.0.CO;2.

889 Dix M, Vohralik P, Bi D, Rashid H, Marsland S et al (2013) The ACCESS coupled model: documentation of core 890 CMIP5 simulations and initial results. Aust Meteorol Ocean 63: 83-99. https://doi.org/10.22499/2.6301.006.

891 Doyle M, Barros V (2002) Midsummer low-level circulation and precipitation in subtropical South America and 892 related sea surface temperature anomalies in the South Atlantic. J Clim 15: 3394-3410. https://doi.org/10.1175/1520893 0442(2002)015<3394:MLLCAP>2.0.CO;2.

894 Dufresne J, Foujols M, Denvil S, Caubel A, Marti O et al (2013) Climate change projections using the IPSL-CM5 895 Earth System Model: from CMIP3 to CMIP5. Clim Dynam 40: 2123-2165. https://doi.org/10.1007/s00382-012896 1636-1.

897 Edson J, Fairall C, Mestayer P, Larsen S (1991) A study of the inertial-dissipation method for computing air-sea 898 fluxes. J Geophys Res 96: 10689-10711. https://doi.org/10.1029/91JC00886. 
Fairall C, Bradley E, Godfrey J, Wick G, Edson J, Youn G (1996) Cool-skin and warm-layer effects on sea surface temperature. J Geophys Res 101: 1295-1308. https://doi.org/10.1029/95JC03190.

Fairall C, Bradley E, Hare J, Grachev A, Edson J (2003) Bulk parameterization of air-sea fluxes: Updates and verification for the COARE algorithm. J Climate 16: 571-591. https://doi.org/10.1175/15200442(2003)016\%3C0571:BPOASF\%3E2.0.CO;2.

Fekete B, Vörösmarty C, Roads J, Willmott C (2004) Uncertainties in precipitation and their impacts on runoff estimates. J Climate 17: 294-304. https://doi.org/10.1175/1520-0442(2004)017\%3C0294:UIPATI\%3E2.0.CO;2.

Feng L, Li J (2006) A comparison of latent heat fluxes over global oceans for ERA andNCEP with GSSTF2. Geophys Res Lett 33: L03810. https://doi.org/10.1029/2005GL024677.

Ferrero M, Villalba R (2019) Interannual and long-term precipitation variability along the subtropical mountains and adjacent Chaco (22-29 S) in Argentina. Front Earth Sci 7: 1-15. https://doi.org/10.3389/feart.2019.00148.

Freitas R, Casagrande F, Lindemann D, Cardoso M, Machado J (2019) The Storm Tracks Response to Changes in Atmospheric Greenhouse Gas Concentration at the South of Brazil and Southwest Atlantic Ocean. Atmos Clim Sci 9: 545-557. https://doi.org/10.4236/acs.2019.94035.

Gan M, Rao B (1991) Surface Cyclogenesis over South America. Mon Weather Rev 119: 1293-1302. https://doi.org/ 10.1175/1520-0493(1991)119<1293:SCOSA>2.0.CO;2.

Gao S, Chiu L, Shie C (2013) Trends and variations of ocean surface latent heatflux: Resultsfrom GSSTF2c data set. Geophys Res Lett 40: 380-385. https://doi.org/10.1029/2012GL054620.

Gramcianinov C, Hodges K, Camargo R (2019) The properties and genesis environments of South Atlantic cyclones. Clim Dynam 53: 4115-4140. https://doi.org/10.1007/s00382-019-04778-1.

Grodsky S, Bentamy A, Carton J, Pinker R (2009) Intraseasonal latent heat flux based on satellite observations. J Climate 22: 4539-4556. https://doi.org/10.1175/2009JCLI2901.1.

Hersbach H, Bell B, Berrisford P, Hirahara S, Horányi A et al (2020) The ERA5 global reanalysis. Q J Roy Meteor Soc 146: 1999-2049. https://doi.org/10.1002/qj.3803.

Hoskins B, Hodges K (2005) A new perspective on Southern Hemisphere storm tracks. J Climate 18: 4108-4129. https://doi.org/10.1175/JCLI3570.1.

Josey S, Gulev S, Yu L (2008). Exchanges through the ocean surface. In: Steele J, Encyclopedia of Ocean Sciences, 2nd. ed., p. 115-140.

Kalnay E, Kanamitsu M, Kistler R, Collins W, Deaven D et al (1996). The NCEP/NCAR 40-Year Reanalysis Project. B Am Meteorol Soc 77: 437-471. https://doi.org/10.1175/1520-0477(1996)077\%3C0437:TNYRP \%3E2.0.CO;2.

Kirtman B, Bitz C, Bryan F, Collins W, Dennis J et al (2012) Impact of ocean model resolution on CCSM climate simulations. Clim Dynam 39: 1303-1328. https://doi.org/10.1007/s00382-012-1500-3.

Kumar B, Cronin M, Joseph S, Ravichandran M, Sureshkumar N (2017) Latent Heat Flux Sensitivity to Sea Surface Temperature: Regional Perspectives. Journal of Climate 30: 129-143. https://doi.org/10.1175/JCLI-D-16-0285.1.

L'Ecuyer T, Beaudoing H, Rodell M, Olson W, Lin B et al (2015) The Observed State of the Energy Budget in the Early Twenty-First Century. J Climate 28: 8319-8346. https://doi.org/10.1175/JCLI-D-14-00556.1.

Legates D, McCabe Junior G (1999) Evaluating the use of "goodness-of-fit" measures in hydrologic and hydroclimatic model validation. Water Resour Res 35: 233-241. https://doi.org/10.1029/1998WR900018.

Leyba I, Solman S, Saraceno M (2019) Trends in sea surface temperature and air-sea heat fluxes over the South Atlantic Ocean. Clim Dynam 53: 4141-4153 https://doi.org/10.1007/s00382-019-04777-2.

Li G, Ren B, Zheng J, Yang C (2011) Net air-sea surface heat flux during 1984-2004 over the North Pacific and North Atlantic oceans $\left(10^{\circ} \mathrm{N}-50^{\circ} \mathrm{N}\right)$ : Annual mean climatology and trend. Theor Appl Climatol 104: 387-401. https://doi.org/10.1007/s00704-010-0351-2. 
Liang X, Yu L (2016) Variations of the Global Net Air-Sea Heat Flux during the "Hiatus”Period (2001-10). J Climate 29: 3647-3660. https://doi.org/10.1175/JCLI-D-15-0626.1.

Liu J, Curry J (2006) Variability of the tropical and subtropical ocean surface latent heat flux during 1989-2000. Geophys Res Lett 33: L05706, doi: 10.1029/2005GL024809.

Liu J, Curry J, Clayson C, Bourassa M (2011) High-Resolution satellite surface latent heat fluxes in North Atlantic hurricanes. Mon Weather Rev 139: 2735-2747. https://doi.org/10.1175/2011MWR3548.1.

Lorenz E (1956) Empirical orthogonal functions and statistical weather prediction. Science Report, Statistical Forecasting Project, Department of Meteorology, MIT (NTIS AD 110268), 49 pp.

Martin G, Bellouin N, Collins W, Culverwell I, Halloran et al (2011) The HadGEM2 family of Met Office Unified Model climate configuration. Geosci Model Dev 4: 723-757. https://doi.org/10.5194/gmd-4-723-2011.

Miysaka T, Nakamura H (2010) Structure and Mechanisms of the Southern Hemisphere Summertime Subtropical Anticyclones. J Climate 23: 2115-2130. https://doi.org/10.1175/2009JCLI3008.1.

Montini T, Jones C, Carvalho L (2019) The South American Low-Level Jet: A New Climatology, Variability, and Changes. J Geophys Res Atmos 124: 1200-1218. https://doi.org/10.1029/2018JD029634.

Moriasi D, Arnold J, Van Liew M, Bingner R, Harmel R, Veith T (2007) Model e Valuation Guidelines for Systematic Quantification of Accuracy in Watershed Simulations. Trans ASABE 50: 885-900. doi: 10.13031/2013.23153.

Morrison A, Griffies S, Winton M, Anderson W, Sarmiento J (2016) Mechanisms of Southern Ocean heat uptake and transport in a global eddying climate model. J Climate 29: 2059-2075. https://doi.org/10.1175/JCLI-D-150579.1.

Nkwinkwa A, Rouault M, Johannessen J (2019) Latent Heat Flux in the Agulhas Current. Remote Sens 11: http://dx.doi.org/10.3390/rs11131576.

Onogi K, Koide H, Sakamoto M, Kobayashi S, Hatsushika H et al (2007) The JRA-25 reanalysis. J Meteorol Soc Jpn 85: 369-432. https://doi.org/10.2151/jmsj.85.369.

Pezzi L, Souza R, Dourado M, Garcia C, Mata M, Silva-Dias M (2005) Ocean-atmosphere in situ observations at the Brazil-Malvinas Confluence region. Geophys Res Lett 32: L22603. https://doi.org/10.1029/2005GL023866.

Pezzi L, Souza R, Acevedo O, Wainer I, Mata M, Garcia C, Camargo R (2009) Multiyear measurements of the oceanic and atmospheric boundary layers at the Brazil-Malvinas confluence region. J Geophys Res-Atmos 114: D19103. https://doi.org/10.1029/2008JD011379.

Pezzi L, Souza R, Farias P, Acevedo O, Miller A (2016) Air-sea interaction at the Southern Brazilian Continental Shelf: In situ observations. J Geophys Res-Oceans 121. https://doi.org/10.1002/2016JC011774.

Pinker R, Bentamy A, Katsaros K, Ma Y, Li C (2014) Estimates of net heat fluxes over the Atlantic Ocean. J Geophys Res-Oceans 119: 410-427. https://doi.org/10.1002/2013JC009386.

Reboita M, Ambrizzi T, Silva B, Pinheiro R, Rocha R (2019) The South Atlantic Subtropical Anticyclone: Present and Future Climate. Front Earth Sci 7. https://doi.org/10.3389/feart.2019.00008.

Roberts M, Hewitt H, Hyder P, Ferreira D, Josey S, Mizielinski M, Shelly A (2016) Impact of ocean resolution on coupled air-sea fluxes and large-scale climate. Geophys Res Lett 43: 430-438. https://doi.org/10.1002/2016GL070559.

Rodell M, Beaudoing H, L'Ecuyer T, Olson W, Famiglietti J et al (2015) The Observed State of the Water Cycle in the Early Twenty-First Century. J Climate 28: 8289-8318. https://doi.org/10.1175/JCLI-D-14-00555.1.

Rotstayn L, Jeffrey S, Collier M, Dravitziki S, Hirst A, Syktus J, Wong K (2012) Aerosol- and greenhouse gasinduced changes in summer rainfall and circulation in the Australasian region: a study using single-forcing climate simulations. Atmos Chem Phys 12: 6377-6404. https://doi.org/10.5194/acp-12-6377-2012.

Saha S, Moorthi S, Pan H, Wu X, Wang J et al (2010) The NCEP climate forecast system reanalysis. B Am Meteorol Soc 91: 1015-1057. https://doi.org/10.1175/2010BAMS3001.1. 
Santhi C, Arnold J, Williams J, Dugas W, Srinivasan R, Hauck L (2001) Validation of the SWAT model on a large river basin with point and nonpoint sources. J Am Water Resour As 37: 1169-1188. https://doi.org/10.1111/j.17521688.2001.tb03630.x.

Santini M, Souza R, Pezzi L, Swart S (2020) Observations of air-sea heat fluxes in the southwestern Atlantic under high-frequency ocean and atmospheric perturbations. Q J Roy Meteor Soc 146: 4226-4251. https://doi.org/10.1002/qj.3905.

Schmidt G, Kelley M, Nazarenko L, Ruedy R, Russell G et al (2014) Configuration and assessment of the GISS ModelE2contributions to the CMIP5 archive. J Adv Model Earth Sy 6: 141-184. https://doi.org/10.1002/2013MS000265.

Singh J, Knapp H, Demissie M (2004) Hydrologic modeling of the Iroquois River watershed using HSPF and SWAT. ISWS CR 2004-08. Champaign, Ill.: Illinois State Water Survey. Available at: www.sws.uiuc.edu/pubdoc/CR/.

Small R, deSzoeke S, Xie S, O’Neill L, Seo H, Song Q, Cornillon P, Spall M, Minobe S (2008) Air-sea interaction over ocean fronts and eddies. Dynam Atmos Oceans 45: 274-319. https://doi.org/10.1016/j.dynatmoce.2008.01.001.

Smith S, Hughes P, Bourassa M (2011) A comparison of nine monthly air-sea flux products. Int J Climatol 31: 1002-1027. https://doi.org/10.1002/joc.2225.

Souza R, Pezzi L (2020) Processos regionais de interação oceano-atmosfera no Atlântico Sul. In: Lana, P.C. and Castello, J.P. Fronteiras do conhecimento em ciência do mar. Ed. FURG, 379p.

Sun X, Cook K, Vizy E (2017) The South Atlantic Subtropical High: Climatology and Interannual Variability. J Climate 30: 3279-3296. https://doi.org/10.1175/JCLI-D-16-0705.1.

Swart S, Gille S, Delille B, Josey S, Mazloff M et al (2019) Constraining Southern Ocean Air-Sea-Ice Fluxes Through Enhanced Observations. Front Mar Sci 6. https://doi.org/10.3389/fmars.2019.00421.

Taylor K, Stouffer R, Meehl G (2012) An overview of CMIP5 and the experiment design. B Am Meteorol Soc 93: 485-498. https://doi.org/10.1175/BAMS-D-11-00094.1.

Tokinaga H, Tanimoto Y, Xie S (2005). SST-induced surface wind variations over the Brazil-Malvinas confluence: Satellite and in situ observations. J Climate 18: 3470-3482. https://doi.org/10.1175/JCLI3485.1.

Trenberth K, Fasullo J, Kiehl J (2009) Earth's global energy budget. B Am Meteorol Soc 90: 311-323. https://doi.org/10.1175/2008BAMS2634.1.

Valdivieso M, Haines K, Balmaseda M, Chang Y, Drevillon M et al (2017) An assessment of air-sea heat fluxes from ocean and coupled reanalyses. Clim Dynam 49: 983-1008. https://doi.org/10.1007/s00382-015-2843-3.

Van Liew M, Arnold J, Garbrecht J (2003) Hydrologic Simulation on Agricultural Watersheds: Choosing Between two Models. Trans ASABE, 46: 1539-1551. doi: 10.13031/2013.15643.

Villas Bôas A, Sato O, Chaigneau A, Castelão G (2015) The signature of mesoscale eddies on the air-sea turbulent heat fluxes in the South Atlantic Ocean. Geophys Res Lett 42: 1856-1862. https://doi.org/10.1002/2015GL063105.

Voldoire A, Sanchez-Gomez E, Salas y Mélia D, Decharme B, Cassou C et al (2012) The CNRM-CM5.1 global climate model: description and basic evaluation. Clim Dynam 40: 2091-2121. https://doi.org/10.1007/s00382-0111259-y.

Volodin E, Diansky N, Gusev A (2013) Simulation and Prediction of Climate Changes in the 19thto 21st Centuries with the Institute of Numerical Mathematics, Russian Academy of Sciences, Model of the Earth's Climate System. Atmos Ocean Phys 49: 347-366. https://doi.org/10.1134/S0001433813040105.

Watanabe M, Suzuki T, O’ishi R, Komuro Y, Watanabe S et al (2010) Improved Climate Simulation by MIROC5: Mean States, Variability, and Climate Sensitivity. J Climate, 23, 6312-6335. https://doi.org/10.1175/2010JCLI3679.1.

Wilks D (2011) Statistical Methods in the Atmospheric Sciences. $3^{\circ}$ ed, Academic Press, 704 pp. 
1032 Willmott C, Matsuura K (2005) Advantages of the mean absolute error (MAE) over the root mean square error 1033 (RMSE) in assessing average model performance. Clim Res 30: 79-82. doi:10.3354/cr030079.

1034 Xu Z, Chang P, Richter I, Kim W, Tang G (2014) Diagnosing southeast tropical Atlantic SST and ocean circulation 1035 biases in the CMIP5 ensemble. Clim Dynam 43: 3123-3145. https://doi.org/10.1007/s00382-014-2247-9.

1036 You Q, Jiang Z, Wang D, Pepin N, Kang S (2017) Simulation of temperature extremes in the Tibetan Plateau from 1037 CMIP5 models and comparison with gridded observations. Clim Dynam 51: 355-369. 1038 https://doi.org/10.1007/s00382-017-3928-y.

1039 Yu L, Weller R, Sun B (2004) Improving Latent and Sensible Heat Flux Estimates for the Atlantic Ocean (1988-99)

1040 by a Synthesis Approach. J Climate 17: 373-393. https://doi.org/10.1175/1520-0442(2004)017\%3C0373:ILASHF 1041 \%3E2.0.CO;2.

1042 Yu L, Jin X, Weller R (2007) Annual, Seasonal, and Interannual Variability of Air-Sea Heat Fluxes in the Indian 1043 Ocean. J Climate 20: 3190-3209. https://doi.org/10.1175/JCLI4163.1.

1044 Yu L, Weller R (2007) Objectively analyzed air-sea heat fluxes for the global ocean-free oceans (1981-2005). B Am 1045 Meteorol Soc 88: 527-539. https://doi.org/10.1175/BAMS-88-4-527.

1046 Yu L, Jin X, Weller R (2008) Multidecade Global Flux Datasets From the Objectively Analyzed Air-Sea Fluxes 1047 (OAFLUX) Project: Latent and Sensible Heat Fluxes, Ocean Evaporation, and Related Surface Meteorological 1048 Variables. Technical Report. OA-2008-01, Woods Hole Oceanographic Institution, OAFLUX Project Woods Hole, 1049 Massachusetts, 64 pp.

1050 Yu L, Zhang Z, Zhong Z, Zhou M, Gao Z, Wu H, Sun B (2011) An inter-comparison of six latent and sensible heat 1051 flux products over the Southern Ocean. Polar Res 30. https://doi.org/10.3402/polar.v30i0.10167.

1052 Zhang R, Wang X, Wang C (2018) On the Simulations of Global Oceanic Latent Heat Flux in the CMIP5 1053 Multimodel Ensemble. J Climate 31: 7111-7128. https://doi.org/10.1175/JCLI-D-17-0713.1. 


\section{Figures}

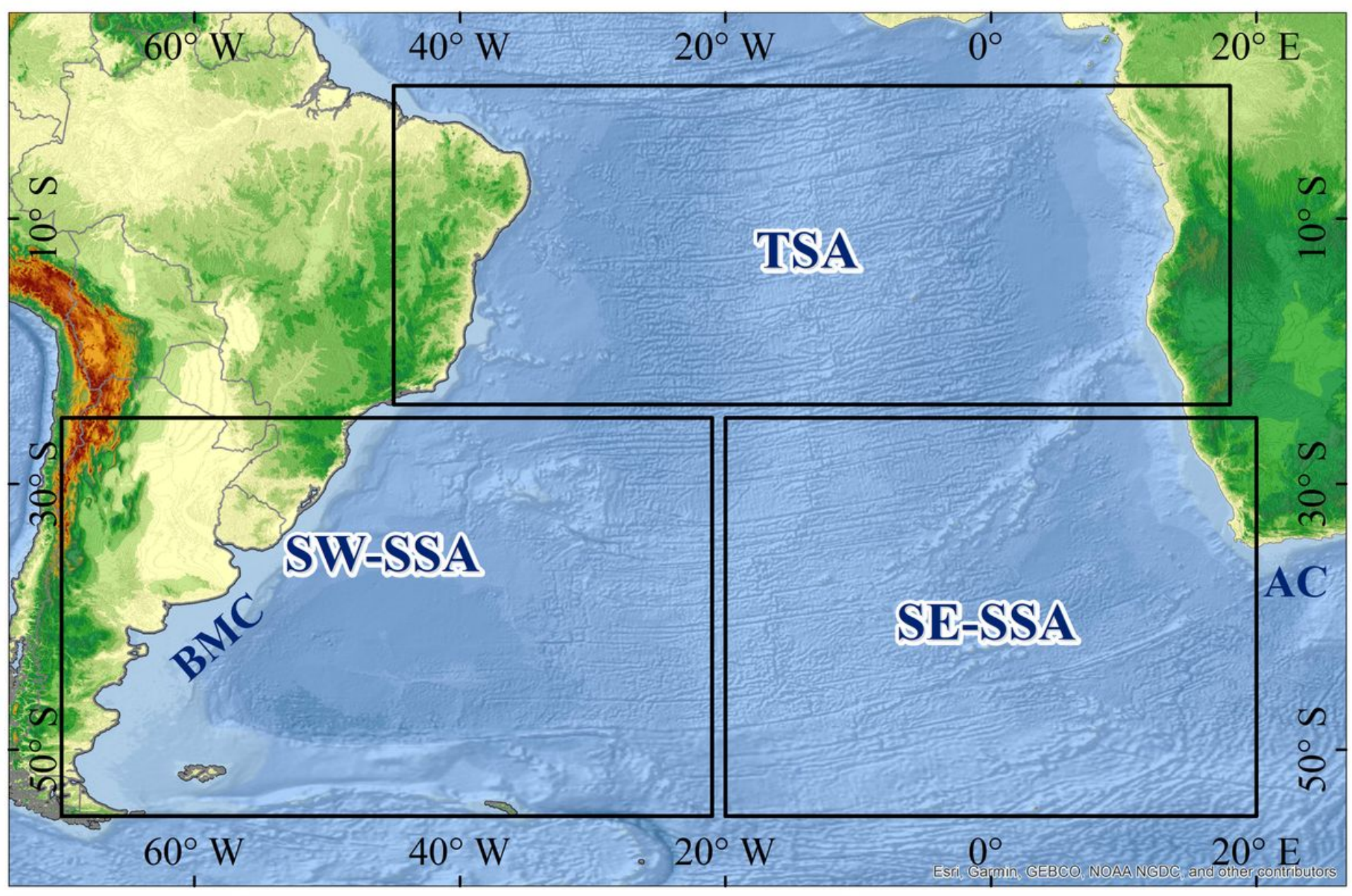

\begin{tabular}{|c|c|c|}
\hline \multirow{6}{*}{$\begin{array}{l}\text { Elevation } \\
\text { m a.s.l. } \\
6795\end{array}$} & Acronym & Name \\
\hline & TSA & Tropical South Atlantic \\
\hline & SE-SSA & Southeastern Subtropical Atlantic \\
\hline & SW-SSA & Southwestern Subtropical Atlantic \\
\hline & $\mathrm{BMC}$ & Brazil-Malvinas Confluence \\
\hline & $\mathrm{AC}$ & Agulhas Current \\
\hline
\end{tabular}

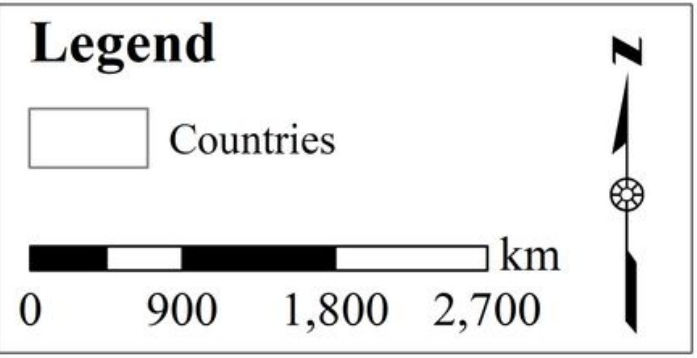

\section{Figure 1}

area of interest in this study is the South Atlantic Ocean (SAO). The area was split into three regions: Southwestern Subtropical Atlantic (SW-SSA); Southeastern Subtropical Atlantic (SE-SSA); and Tropical South Atlantic (TSA). Note: The designations employed and the presentation of the material on this map do not imply the expression of any opinion whatsoever on the part of Research Square concerning the legal status of any country, territory, city or area or of its authorities, or concerning the delimitation of its frontiers or boundaries. This map has been provided by the authors. 


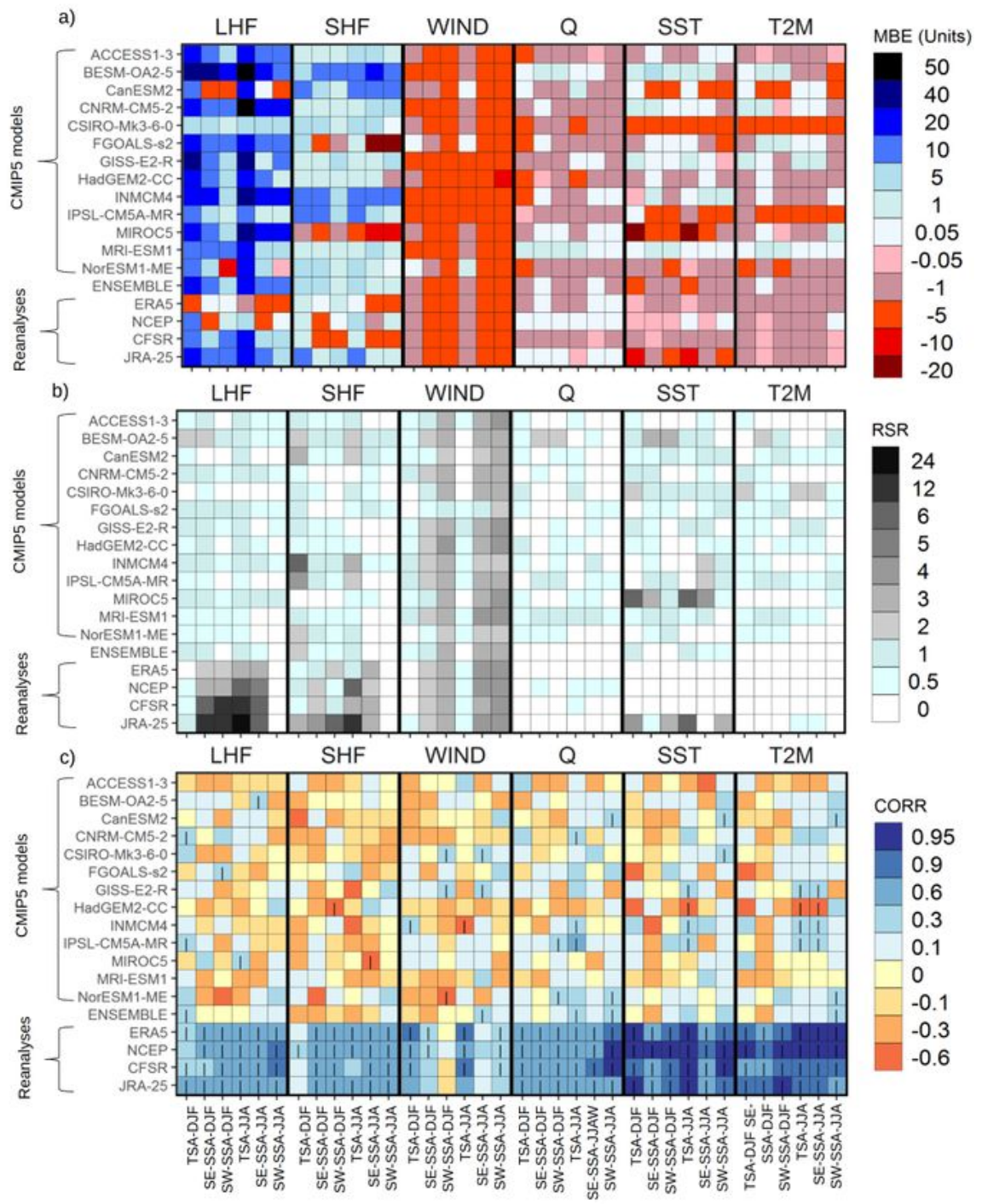

Figure 2

Statistical tests for the variables under analysis for each SAO region (TSA, SE-SSA, and SW-SSA) during the two seasonal periods (DJF and JJA); for the thirteen models from CMIP5, the Ensemble (MME) and the four reanalyzes compared with the observational dataset - OAFlux: a) BIAS (top panel); b) the RSR (central panel); and c) the CORR (bottom panel). The black line on the CORR indicates that the correlation is significant at $95 \%$. 


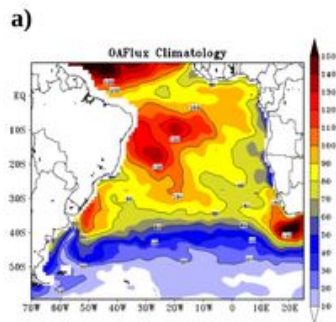

b)

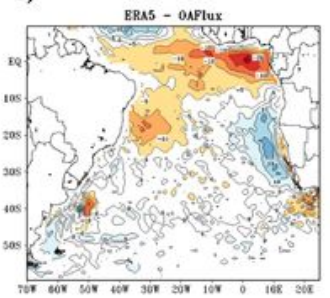

d)

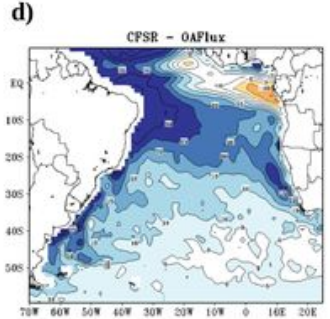

g)

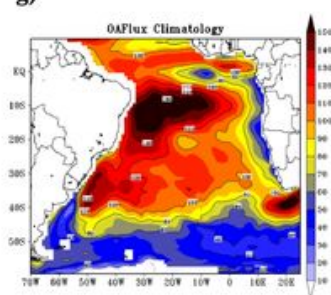

j)

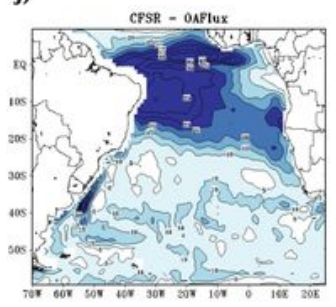

c)

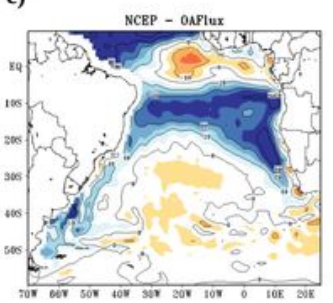

f)

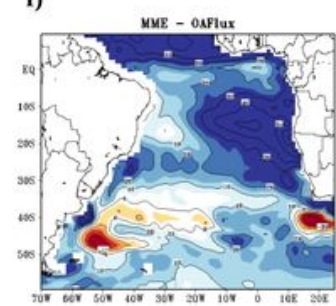

i)

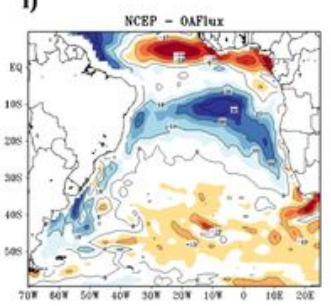

l)

k)

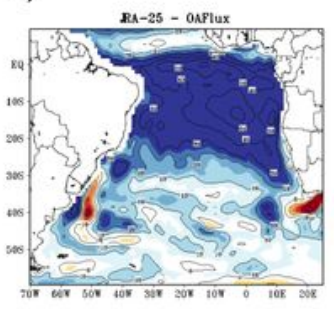

h)
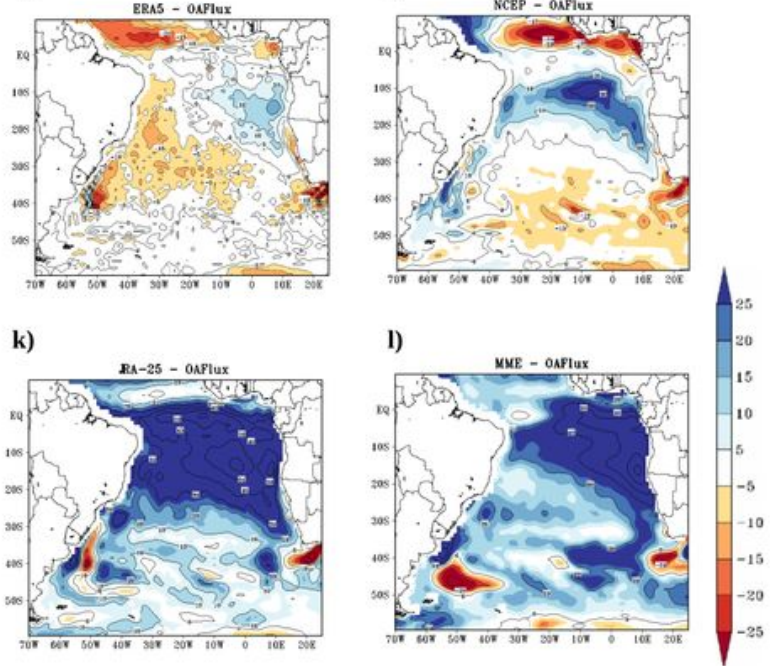

Figure 3

Latent heat flux (LHF) in W.m-2 from 1980 to 2005, DJF (a-f) and JJA (g-m): a-g) OAFLux climatology; MBE calculation from datasets and OAFlux: b-h) ERA5; c-i) NCEP, d-j) CFSR, e-k) JRA-25; and f-l) MME Note: The designations employed and the presentation of the material on this map do not imply the expression of any opinion whatsoever on the part of Research Square concerning the legal status of any 
country, territory, city or area or of its authorities, or concerning the delimitation of its frontiers or boundaries. This map has been provided by the authors.
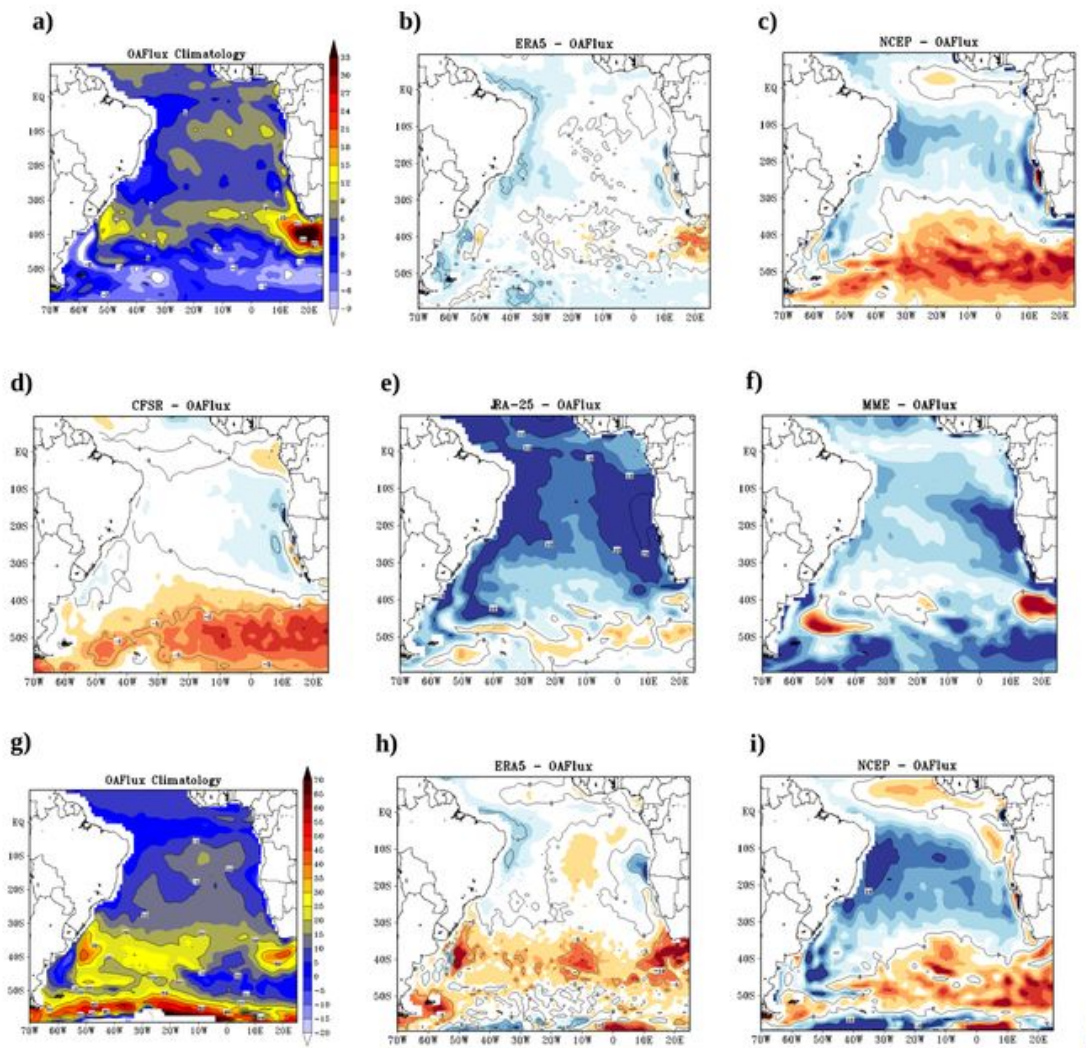

h)
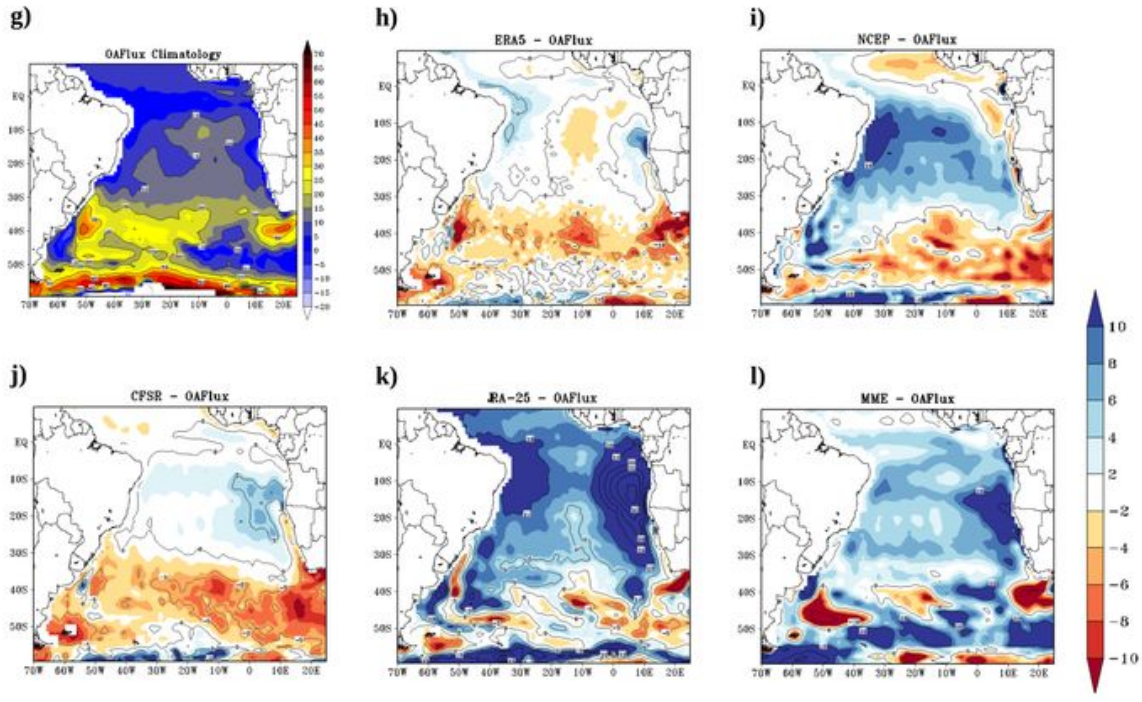

\section{Figure 4}

Latent heat flux (LHF), in W.m-2, from 1980 to 2005, DJF (a-f) and JJA (g-m): a-g) climatology of OAFLux; calculation of MBE between datasets and OAFlux: b-h) ERA5; c-i) NCEP, d-j) CFSR, e-k) JRA-25; and f-I) MME Note: The designations employed and the presentation of the material on this map do not imply the 
expression of any opinion whatsoever on the part of Research Square concerning the legal status of any country, territory, city or area or of its authorities, or concerning the delimitation of its frontiers or boundaries. This map has been provided by the authors.
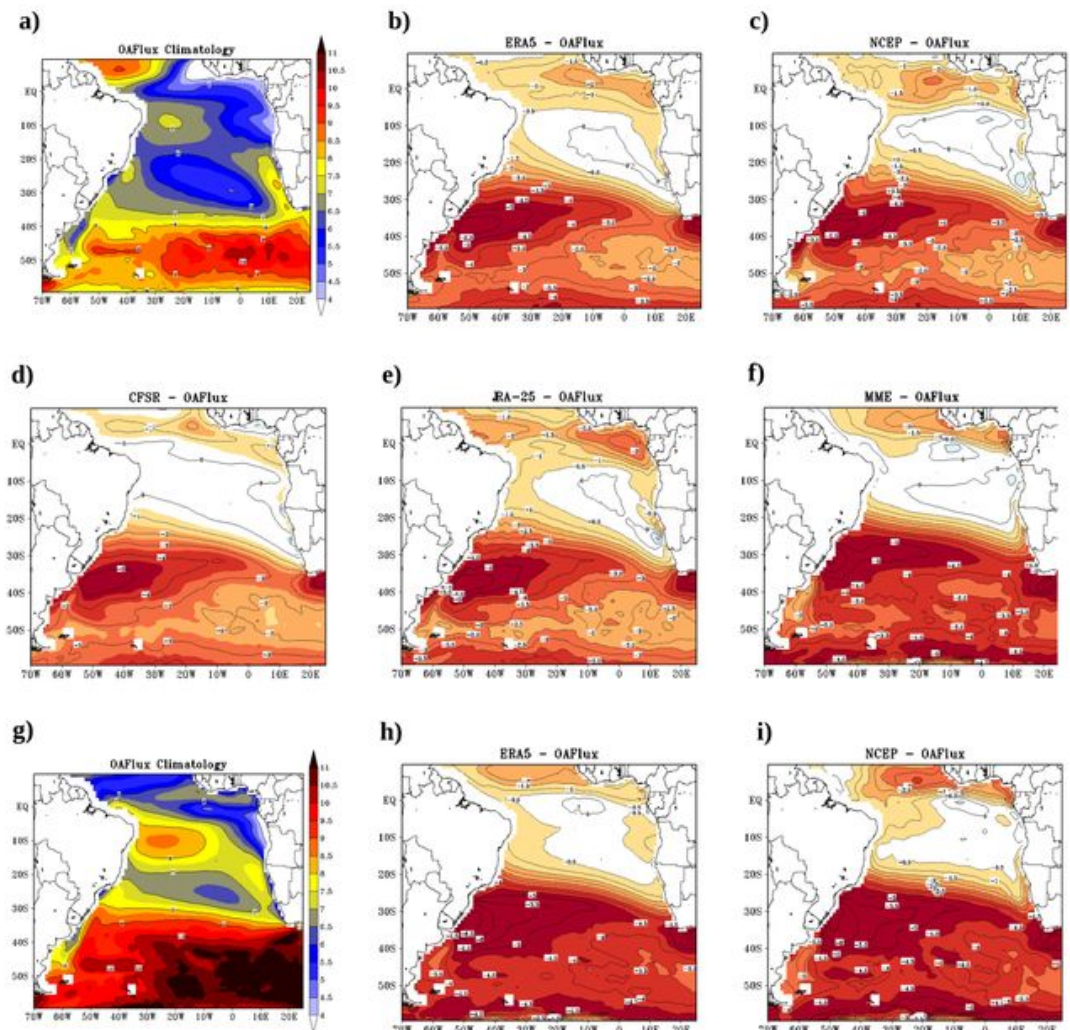

h)
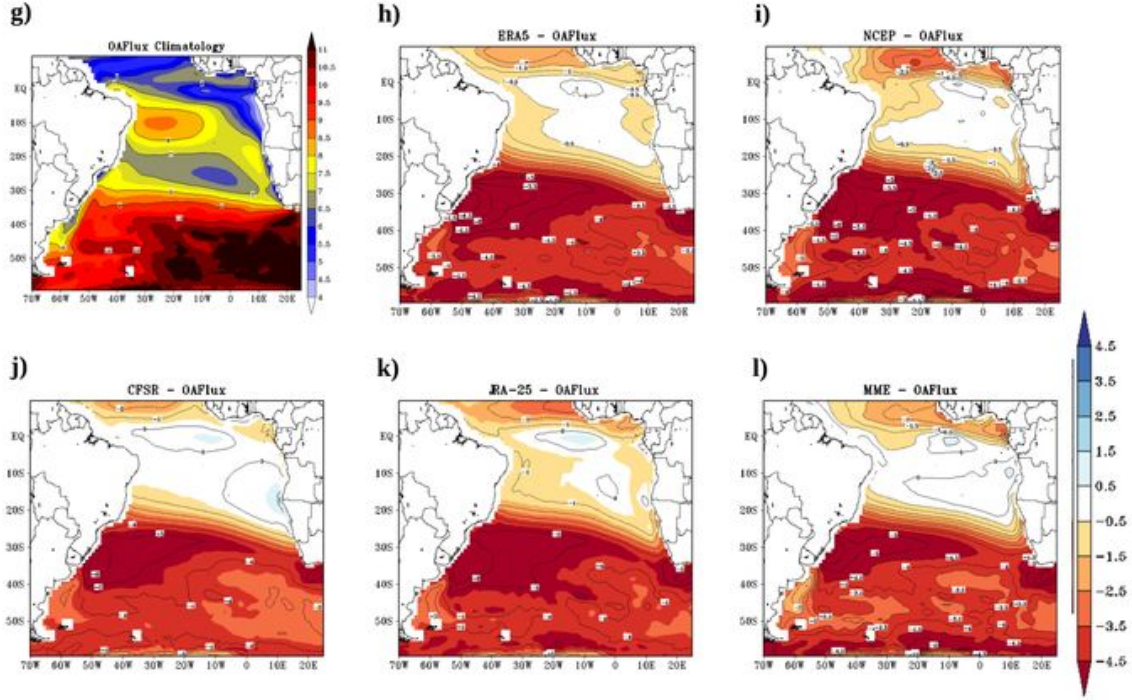

\section{Figure 5}

Wind speed (WIND), in m.s-1, from 1980 to 2005, DJF (a-f) and JJA (g-m): a-g) OAFLux climatology; calculation of MBE between datasets and OAFlux: $b-h$ ) ERA5; c-i) NCEP, d-j) CFSR, e-k) JRA-25; and f-I) 
MME. Note: The designations employed and the presentation of the material on this map do not imply the expression of any opinion whatsoever on the part of Research Square concerning the legal status of any country, territory, city or area or of its authorities, or concerning the delimitation of its frontiers or boundaries. This map has been provided by the authors.
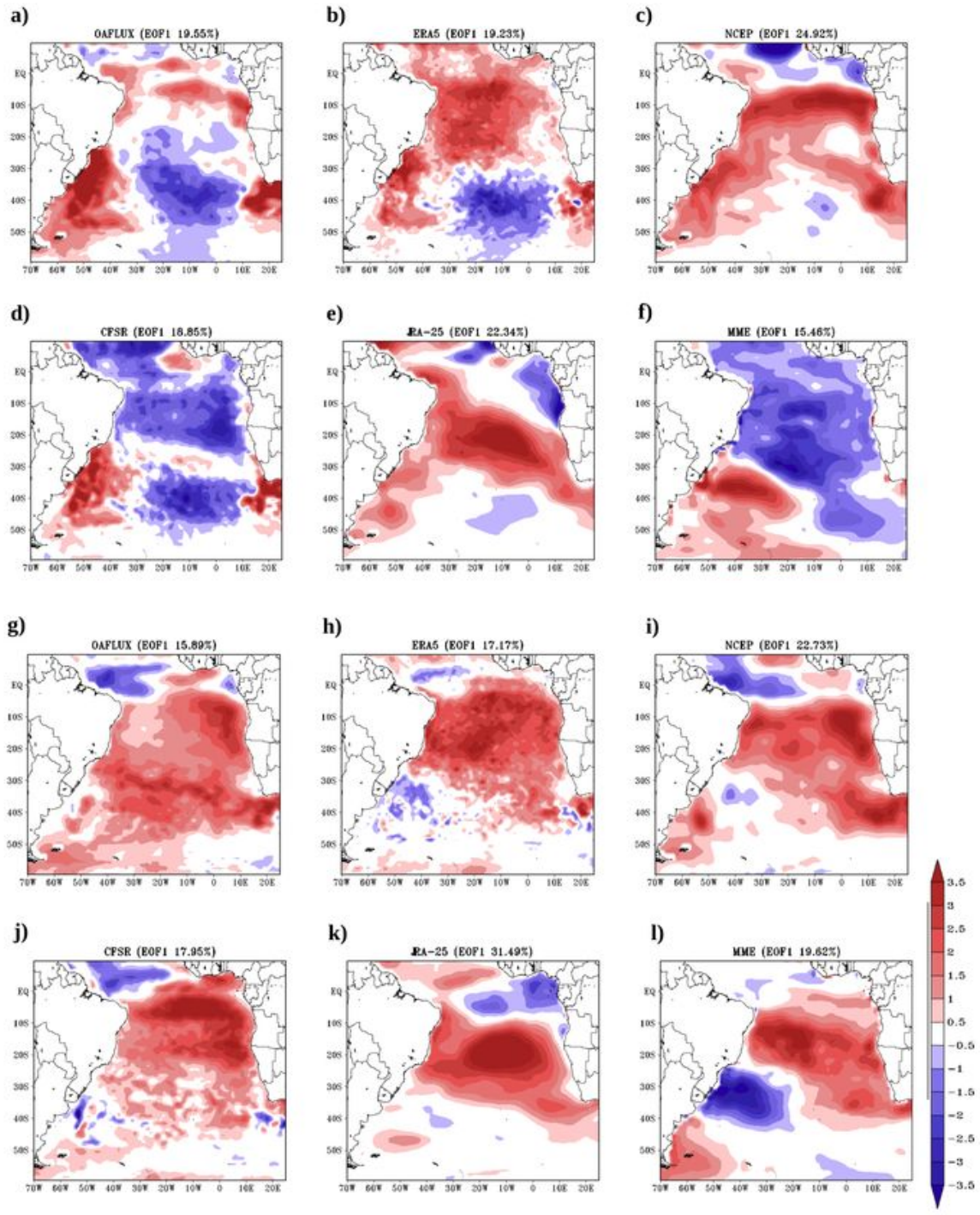

Figure 6 
Seasonal spatial loading patterns of the first LHF mode computed for the 1980-2005 period in the south Atlantic Ocean, for DJF (a-f) and JJA (g-m): a-g) OAFLux; b-h) ERA5; c-i) NCEP, d-j) CFSR, e-k) JRA-25; and $\mathrm{f}-\mathrm{I}) \mathrm{MME}$. Note: The designations employed and the presentation of the material on this map do not imply the expression of any opinion whatsoever on the part of Research Square concerning the legal status of any country, territory, city or area or of its authorities, or concerning the delimitation of its frontiers or boundaries. This map has been provided by the authors.

a)

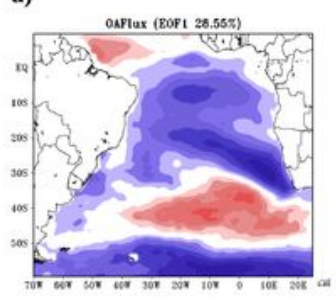

d)

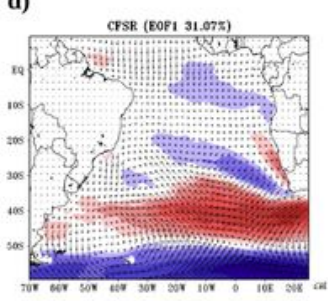

g)

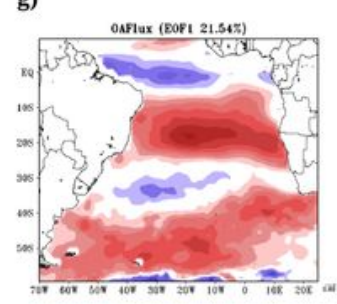

j)

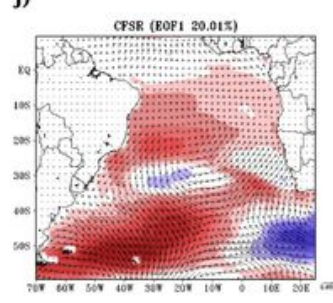

b)

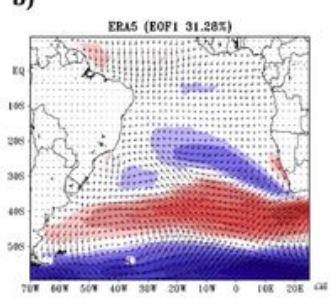

e)

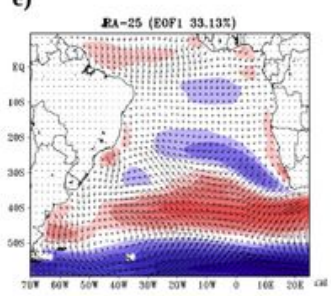

h)

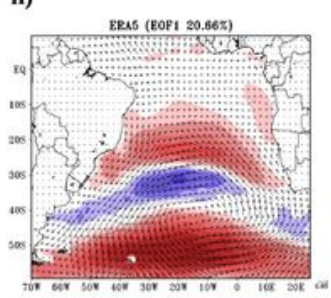

k)

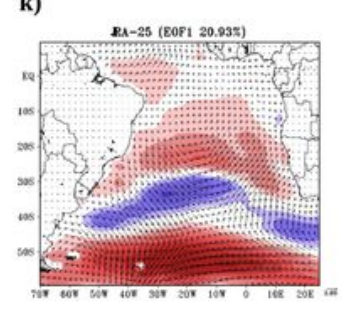

c)

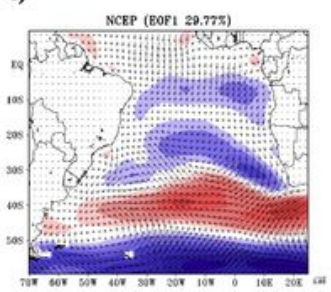

f)

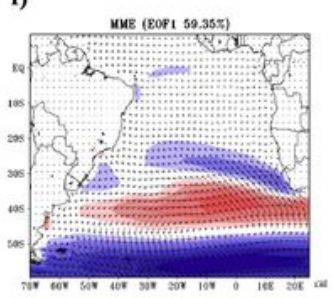

i)

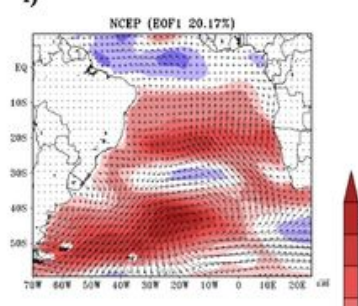

l)

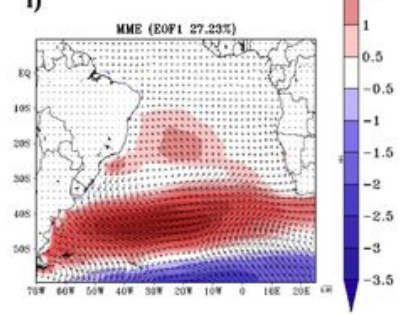


First Principal Component of EOF for WIND, from 1980 to 2005, for DJF (a-f) and JJA (g-m): a-g) OAFLux; b-h) ERA5; c-i) NCEP, d-j) CFSR, e-k) JRA-25; and f-I) MME. Note: The designations employed and the presentation of the material on this map do not imply the expression of any opinion whatsoever on the part of Research Square concerning the legal status of any country, territory, city or area or of its authorities, or concerning the delimitation of its frontiers or boundaries. This map has been provided by the authors.

\section{Supplementary Files}

This is a list of supplementary files associated with this preprint. Click to download.

- Table1.pdf

- Table2.pdf 